\title{
Article
}

\section{An investigation of expertise in cycling: Eye tracking, Think Aloud and the influence of a competitor}

Massey, Hollie, Whitehead, Amy, Marchant, David, Polman, Remco and Williams, Emily L.

Available at http://clok.uclan.ac.uk/33125/

Massey, Hollie ORCID: 0000-0002-9793-8702, Whitehead, Amy, Marchant, David, Polman, Remco and Williams, Emily L. (2020) An investigation of expertise in cycling: Eye tracking, Think Aloud and the influence of a competitor. Psychology of Sport and Exercise, 49 . ISSN 1469-0292

It is advisable to refer to the publisher's version if you intend to cite from the work. http://dx.doi.org/10.1016/j.psychsport.2020.101681

For more information about UCLan's research in this area go to http://www.uclan.ac.uk/researchgroups/ and search for <name of research Group>.

For information about Research generally at UCLan please go to http://www.uclan.ac.uk/research/

All outputs in CLoK are protected by Intellectual Property Rights law, including Copyright law. Copyright, IPR and Moral Rights for the works on this site are retained by the individual authors and/or other copyright owners. Terms and conditions for use of this material are defined in the policies page.

\section{CLoK}

Central Lancashire online Knowledge www.clok.uclan.ac.uk

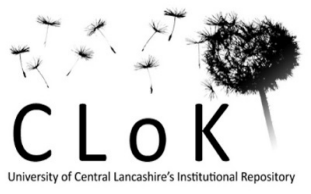


1 An investigation of expertise in cycling: Eye tracking, Think Aloud and the influence of a

2

3

4

5

6 Hollie S Massey ${ }^{\mathrm{a}}$, Amy E Whitehead ${ }^{\mathrm{b}}$, David Marchant ${ }^{\mathrm{c}}$, Remco C Polman ${ }^{\mathrm{d}}$ Emily L Williams $^{\mathrm{e}}$

7

8

9

10

11

12

13

14

15

16

17

18

19

20

21

22

23

24

25

\section{Authors and affiliations}

${ }^{a}$ University of Central Lancashire, School of Psychology,UK. HMassey@uclan.ac.uk

${ }^{b}$ Liverpool John Moores University, School of Sport and Exercise Sciences, UK.

A.E.Whitehead@1jmu.ac.uk

${ }^{c}$ Edge Hill University, Sport and Exercise Science, UK. Marchand@edgehill.ac.uk

${ }^{\mathrm{d}}$ Queensland University of Technology, Exercise and Nutrition Sciences, Australia, remco.polman@qut.edu.au

${ }^{e}$ Leeds Beckett University, School of Sport, UK. Emily.Williams@leedsbeckett.ac.uk

\section{Correspondence:}

Hollie S Massey, University of Central Lancashire, School of Psychology, UK. HMassey@uclan.ac.uk 
Expertise differences in cognitions, gaze behaviour and cycling performance

\section{An investigation of expertise in cycling: Eye tracking, Think Aloud and the influence of a}

competitor

\section{Abstract}

Objectives: Two studies investigated expert-novice differences in information-seeking behaviour, cognitions and performance during cycling time trials (TT). Study 1 examined trained and novice cyclist's cognitions whilst performing a TT, using a Think Aloud (TA) protocol and eye-tracking techniques. Study 2 investigated expertise differences during alone and competitive TTs. Methods: in Study 1, six trained and seven novice cyclists performed a $16.1 \mathrm{~km} \mathrm{TT.} \mathrm{In} \mathrm{Study} \mathrm{2,} \mathrm{eight} \mathrm{trained} \mathrm{and}$ ten novice cyclists performed three $16.1 \mathrm{~km}$ TTs; a baseline TT, an alone TT and a trial against a virtual competitor. In both studies, participants were asked to TA and in Study 1 they also wore mobile gazetracking glasses. Performance feedback and a simulated TT course were visually displayed during all trials, as was a virtual avatar during the competitor trial. Verbalisations were coded into primary and secondary themes. Cognitions and pacing strategies were compared between groups and across the duration of the TTs. In Study 1, eye-tracking data for total dwell time and gaze frequency were calculated for each area of interest (Time Elapsed, Power, Heart Rate, Cadence, Distance Covered, Speed and Course Scenery). Results: In Study 1, no significant differences were found in informationseeking behaviour between groups, however there were expertise differences in the cognitive strategies used. Trained cyclists' verbalisations were more performance-relevant (i.e., power output), whereas the untrained group were more focused on task completion (i.e., distance and time) and irrelevant information. Both groups talked more about distance and motivational thoughts in the later stages of the trial, and dwell time on distance feedback also increased in this final $4 \mathrm{~km}$. In Study 2, the trained group performed faster than the untrained group but there were no significant differences in pace or performance between alone and competitive TTs for either group. Differences in cognitions were found between groups and across the TT duration. Conclusion: Both studies demonstrate that cognitive processes differ as a function of expertise during self-paced cycling time trials. There were 
Expertise differences in cognitions, gaze behaviour and cycling performance

51 no differences in information-seeking behaviour between trained and untrained cyclists and there was

52 no effect of an opponent on pace or performance.

Key Words: Pacing, Cognition, Competition, Gaze Behaviour, Performance, Feedback

54

55

56

57

58

59

60

61

62

63

64

65

66

67

68

69

70

71

72

\section{Introduction}

Athletes develop experience-primed pacing strategies which allow them to complete an endurance event without physical harm whilst equally maximising their goal achievement (Edwards \& Polman, 2012). Following an initially physiology-driven theoretical stance (e.g., Ulmer, 1996; Hill \& Long, 1925), more recent research has presented arguments that cognitions and perceptions explain how pacing strategies are developed, maintained and altered during endurance performance (Marcora, 2008). Specifically, theories of decision-making have been applied to the continuous nature of self-paced exercise and suggest that exertion is regulated by continual cognitive decisions in response to physiological disturbances, perceived levels of effort, performance feedback and psychological drive (Smits, Pepping, \& Hettinga, 2014).

Understanding the cognitive factors that discriminate between experts and novices has been a longstanding focus of research (see Cona et al., 2015). More recently, within the endurance performance field, the different cognitive strategies used by athletes of various training status' (e.g. elite vs recreational) have been explored (McCormick, Meijen, \& Marcora, 2015). Understanding these cognitive differences has widespread application in allowing practitioners to more effectively implement psychological support/interventions to athletes at different performance levels. Therefore, this study aims to further investigate these expert-novice differences in pacing behaviour by examining conscious cognitions, gaze behaviour and the influence of a competitor during endurance performance.

Empirical research brings with it methodological difficulties in exploring the cognitions that underpin decision-making in sport, with previous research mostly reporting retrospective accounts from 
Expertise differences in cognitions, gaze behaviour and cycling performance

athletes (Brick, Campbell, Sheehan, Fitzpatrick, \& Maclntyre, 2018). For example, studies which have examined the metacognitive processes and attentional focus in endurance runners via retrospective interviews (Brick et al., 2018; Brick, Campbell, Metcalfe, Mair, \& Maclntyre, 2015) are limited by memory decay, reporting bias (Whitehead, Taylor, \& Polman, 2015; Nicholls \& Polman, 2008) and the outcome (Bahrick, Hall, \& Berger, 1996). As an alternative, the Think Aloud (TA) protocol allows the capture of the dynamic and complex cognitive processes that underpin decisions, in real time. TA requires individuals to continuously verbalise their thoughts over the duration of a task (Ericsson \& Simon, 1980). A growing body of research has applied TA in endurance events, such as running (Samson, Simpson, Kamphoff, \& Langlier, 2015) and cycling (Whitehead et al., 2017; Whitehead et al., 2018), to capture 'in-event' cognitions. These studies have demonstrated how TA can be a viable method to collect these in-event cognitions and attentional focus. Furthermore, recent evidence demonstrates that cyclists perceive that TA does not affect their performance in either lab or field setting (Whitehead et al., 2018).

Sport TA research has found consistent differences in meta-cognitive expertise. In tennis and golf, more skilled performers engaged in higher levels of planning, whereas lower skilled performers' cognitions were more technical (Whitehead et al., 2015; McPherson \& Kernodle, 2007). Using TA within cycling, Whitehead et al. (2018) found that trained cyclists use active self-regulatory strategies during their performance and maintain a task-relevant focus, whereas inexperienced individuals attempt to use distractive strategies to overcome perceptions of pain and fatigue. It has been suggested that these types of perceptions are also necessary for trained athletes to monitor, and in some instances may even be considered essential in the accomplishment of goals (Bale, 2006; Simpson, Post, Young, \& Jensen, 2014), but those less experienced may only interpret them as negative cues. Theoretically, such findings align with the conscious awareness brain regulation model of pacing (Edwards \& Polman, 2013). This model proposes that exercise is regulated using the athlete's prior experience, knowledge of the exercise endpoint and afferent feedback in which pacing is seen as a decision-making process. Only in instances when the magnitude of the sensory information 
Expertise differences in cognitions, gaze behaviour and cycling performance

101

102

103

104

105

106

107

108

110

111

112

threatens homeostasis does it reach awareness and conscious regulation of the task occurs. In addition, these expertise differences in cognitions allow us to identify how experience influences the type of in-task cognitive strategies and how they may drive decisions to alter pace.

TA is not without its limitations, including the difficulty of assessing unconscious and automated processes. Instead, measures of overt attentional allocation such as eye tracking allow for the unobtrusive capture of information acquisition, which can provide insight into participants' information use during exercise (Boya et al., 2017). Combining TA and eye tracking allows for a novel insight into the interaction between visual and cognitive processes that are occurring during an exercise bout. Specifically, the active and overt efforts to acquire and use information from the visual environment.

Vision is the dominant sensory system underpinning human performance (Williams, Davids, \& Williams, 1999) and has received significant research attention in sporting contexts. Experts effectively and efficiently use the visual system to allocate attention and guide performance compared to novices (Mann, Williams, Ward \& Janelle, 2007). Both the number and duration of fixations indicate an individual's point of interest and relative attention allocation. Longer fixations are thought to facilitate greater information processing (albeit not necessarily from the point of fixation). For overt attentional allocation, the number of fixations provides an indication of the search strategies employed to extract information from the environment. In sporting settings, two separate meta-analyses (Voss, Kramer, Basak, Parkash, \& Roberts, 2010; Mann et al., 2007) support the view that expert performers possess enhanced perceptual-cognitive skills, evidenced through effective attention allocation and cue utilization. Experts extract greater task-relevant information using fewer fixations of longer duration when compared to non-experts who typically utilise fixations of shorter duration (Mann et al., 2007), and this is associated with visual search strategies directed to the most important targets and objects in display (e.g., Vickers, 2007; Williams \& Ford, 2008). However, such findings are balanced by the observation that athletes' gaze behaviours can differ significantly between video simulation and field- 
Expertise differences in cognitions, gaze behaviour and cycling performance

based settings reflecting different task constraints (e.g., Dicks, Davids, \& Button, 2010), with expertise effects more apparent under naturalistic conditions (see Mann et al., 2007).

Using eye tracking technology, recent research has investigated information-acquisition strategies during cycling $T T$ performance and more specifically the differences in this behaviour between expert and novice athletes (Boya et al., 2017). Experienced TT cyclists' attention was directed primarily to speed, with distance feedback a secondary source during a $16.1 \mathrm{~km}$ TT. Novice cyclists exhibited a less consistent pattern of information source usage, but with a trend towards dependence on distance feedback. Furthermore, novices exhibited high frequency glances of shorter duration when compared to experienced cyclists. Such patterns of visual information use reflect previous TA findings during cycling, where novice's cognitions have similarly been found to be more focussed on distance (Whitehead et al., 2018). Whilst highlighting the importance of different information sources, visual fixation does not necessarily imply the use of the information for processing and actioning as it may not represent the locus of attention (Vater, Williams, \& Hossner, 2019). As such, the combination of eye-tracking and TA data collection simultaneously, would provide a richer indication of how pacing decisions are derived during exertional tasks.

In addition to performance feedback, cyclists have also been shown to alter pace and/or perform faster when riding with a virtual pacing avatar in comparison to a baseline, ride-alone trial (Williams, el al. 2014: Corbett, Barwood, Ouzounoglou, Thelwell, \& Dicks, 2012; Stone et al., 2012). This performance enhancement is supported by classic Social Facilitation theory (Triplett, 1898), increases in motivational drive (McCormick et al., 2015), a shift to a more external attentional focus and lowered perceptions of exertion (Williams et al. 2014). Due to the limitations associated with intermittent psychological measures such as RPE, these mechanisms are not yet fully understood.

Furthermore, whilst an awareness of competitors is an important source of information for metacognitive performance regulation (Brisk et al., 2014), their behaviour is interpreted in line with personal capacity (Baker, Côté, \& Deakin, 2005). Decision-making and pacing regulation throughout 
Expertise differences in cognitions, gaze behaviour and cycling performance

151

152

153

154

155

156

157

158

159

160

161

162

163

164

165

166

167

168

169

170

171

172

173

174

an event is largely derived through an athlete's interpretation of their own performance versus the performance of a competitor (Hettinga et al., 2017b). Tactical decisions to alter pacing strategies in response to the behaviour of a competitor must be balanced with affordances of the athlete's physiological capability and psychological drive to achieve an optimal performance (Hettinga, Konings,

\& Pepping, 2017a); the affordance-competition hypothesis (Cisek \& Kalaska, 2010; Cisek, 2007). Konings, Foulsham, Micklewright, and Hettinga (2019) demonstrated that pacing and visual attention are altered when there is a high athlete-opponent interdependency compared to both low interdependence and ride-alone cycling time trials. However, what is less known is how performance data and other external information, when available, inform cognitions and thus pacing decisions. Insight into the processing of competitor-relevant information would provide useful insight into selfregulatory efforts.

Whilst expertise differences exist in cognitive focus during an endurance task (e.g., Whitehead et al., 2018), it is not clear how the presence of a competitor may influence these cognitions. Furthermore, only one known study has investigated differences in visual search strategies between experts and novices in endurance performance using eye tracking (Boya et al., 2017). Therefore, in this manuscript, two studies are presented. Study 1 aimed to investigate gaze behaviour and cognitive differences between trained and untrained cyclists during a $16.1 \mathrm{~km}$ time trial. Study 2 aimed to investigate the influence of a competitor on trained and untrained cyclists' cognitions and performance during 16.1 km time trials.

Study 1: Differences in cognitions between trained and untrained athletes during cycling performance: Utilising eye tracking and Think Aloud techniques

The present study used gaze-tracking technology and TA protocol to investigate changes in cyclists' visual and cognitive behaviour when presented with performance feedback. This study is the first to capture the dynamic and complex nature of these cognitions and behaviours with both novice and trained cyclists. The overall aim of this study was to investigate expert-novice differences in 
Expertise differences in cognitions, gaze behaviour and cycling performance

information seeking behaviour, cognitions and performance during a cycling TT. Secondly, it aimed to identify how these behaviours and cognitions may change over the course of the time trial. It was predicted that experienced cyclists would perform faster, focus more on visual performance feedback and use more task-relevant cognitive strategies compared to novice individuals. Furthermore, it was predicted that cognitions would change over time, with an expected increase in cognitions relating to distance and motivation in the later stages of the trial.

\section{Methods}

\section{Design}

A two-way mixed experimental design compared differences in performance, cognitions and information-seeking behaviour between trained and untrained participants (between-group factor) across distance covered (within-group factor) in a $16.1 \mathrm{~km}$ cycling time trial. Participants attended a single testing session to perform the TT. Performance time (min:s), speed $\left(\mathrm{km} \cdot \mathrm{hr}-{ }^{1}\right)$, power output $(W)$, heart rate (beats. $\mathrm{min}^{-1}$ ), cadence $\left(\right.$ revs. $\mathrm{min}^{-1}$ ) and participants' verbalisations were continuously recorded and eye tracking techniques were used to measure the type, duration and frequency of information that was looked at throughout the $\pi$.

\section{Participants}

Six trained male cyclists $(M$ age $=43.0 \pm 18.8$ years; $M$ height $=173.5 \pm 4.8 \mathrm{~cm} ; M$ body mass $=65.3 \pm$ $2.8 \mathrm{~kg}$ ) and seven untrained, physically active males $(M$ age $=36.4 \pm 5.9$ years; $M$ height $=176.6 \pm 4.1$ $\mathrm{cm} ; M$ body mass $=82.7 \pm 15.5 \mathrm{~kg}$ ) volunteered for the study. The sample size in this study (and Study 2) is comparable to other similar studies in this field of research (e.g., Brick et al., 2018; Whitehead et al., 2018; Boya et al., 2017). Whilst larger sample sizes are recommended, it has been argued that this can compete with other laudable goals of research, including adopting a multi-method design as well as recruiting a targeted group of participants, as seen in the current study (Schweizer \& Furley, 2016). Criteria for the trained participants stipulated a minimum of two years competitive cycling experience, be currently training at least 5 hours and/or $60 \mathrm{~km}$ a week, and have a personal best $16.1 \mathrm{~km}$ road TT 
Expertise differences in cognitions, gaze behaviour and cycling performance

201

202

203

204

205

206

207

208

209

210

211

212

213

214

215

216

217

218

219

221

222

223

time of less than 25 mins achieved in the last three years (de Pauw et al., 2013). Those in the untrained group did not have prior competitive cycling experience but were healthy and physically active, according to government guidelines (i.e. $>150$ minutes of moderate exercise per week). All participants had normal visual acuity either unaided or whilst wearing their own corrective lenses which were worn during the trials. All participants provided written informed consent and ethical approval was granted by the institutional research ethics committee before the study was conducted.

\section{Materials}

Participants performed one $16.1 \mathrm{~km}$ cycling $\Pi \mathrm{T}$ in a laboratory on an electromagnetically-braked cycle ergometer (Velotron Pro, RacerMate, Seattle, USA) that was calibrated in accordance with manufacturer's guidelines. Directly in front of the bike, a $240 \mathrm{~cm}$ by $200 \mathrm{~cm}$ screen was positioned above and parallel to the floor, displaying a simulated TT course using ergometry software (RacerMate software, Version 4.0.2). The cycle ergometer was positioned centrally such that the screen occupied the majority of the participant's field of view, and the perception of the road was a realistic position to enhance the simulative effects of the passing scenery. The visual course was a flat, straight road in a rural outdoor environment. Real-time performance feedback displayed from left to right horizontally across the bottom segment of the screen was speed $\left(\mathrm{km} \cdot \mathrm{hr}^{-1}\right)$, distance covered $(\mathrm{km})$, cadence (r.min $\left.{ }^{1}\right)$, power output (W), and heart rate (bpm). Time elapsed (min: sec) was presented above the heart rate feedback. A simulated, dynamic avatar was projected on the TT road representing the participants' speed profile throughout. Participants were fitted with a Polar heart rate monitor (Polar Team System, Polar Electro, Kempele, Finland) which recorded heart rate at a $5 \mathrm{~s}$ sampling rate and was integrated with the ergometry software.

Eye movements were recorded during each TT with lightweight $(70 \mathrm{~g})$ wearable mobile gaze-tracking glasses (SensoMotoric Instruments Eye Tracking Glasses 2 Wireless, SMI ETG) at a sampling rate of $30 \mathrm{~Hz}$. This binocular eye-tracking system contains two cameras directed towards participants' eyes and projects infrared light through six LEDs directed at each eye to record eye movements. It has an 
Expertise differences in cognitions, gaze behaviour and cycling performance

automatic parallax compensation, a spatial resolution of 0.1 degrees and a gaze position accuracy of 0.5 degrees across all distances. The glasses are equipped with a HD scene camera (resolution $1280 \mathrm{x}$ $960 \mathrm{px}$ ) with light settings adjusted according to the indoor environment. Glasses were calibrated prior to each $\Pi T$ using a three-point calibration system on a five-point grid displayed on-screen in front of the participant. The glasses were connected to a mobile recording unit, secured in a buckled waist belt worn by the participants. The integrated microphone in the glasses was also used to record participants' verbalisations throughout the TT and the all data were recorded using the iViewETG software.

\section{Procedure}

Prior to the testing session, participants were instructed not to consume alcohol or participate in strenuous physical activity in the 24 hours before. Caffeine ingestion and food were not permitted in the preceding 2 hours and they were asked to consume at least $500 \mathrm{ml}$ of water during these 2 hours. Upon arrival, measurements of height and body mass were taken.

\section{Cycle Ergometry}

After describing the nature of the trial and visual feedback that would be provided, the cycle ergometer was adjusted to the participant's stature and preferences. A 10-minute self-paced warmup was then performed where participants maintained a heart rate approximate to $70 \%$ of their theoretical maximal heart rate (220-age). A three-minute rest period was provided prior to commencing the TT and participants were reminded that they should complete the trial in the fastest time possible and exert maximal effort. During the $\Pi$, water was consumed ad libitum but no other fluids or nutritional intake was permitted. A standing fan was used at the participants' discretion and a self-determined warm down was completed. 
Expertise differences in cognitions, gaze behaviour and cycling performance

Prior to testing, detailed instructions were provided explaining the TA protocol. Participants undertook a series of TA training exercises adapted from Ericsson and Kirk (2001) and also practiced thinking aloud during training sessions (trained group) or physical activity (untrained group) in the week prior to testing. The TA training exercises used Ericsson and Kirk's (2001) adapted directions for giving TA verbal reports, which included providing verbal reports during the warm-up task and completing non-cycling problems; (1) an alphabet exercise, (2) counting the number of dots on a page, and (3) verbal recall.

Upon arrival to the laboratory, participants confirmed their engagement with the training exercises before TA instructions were reiterated. Participants were instructed to use Level 2 TA and were asked to "please Think Aloud by trying to say out loud anything that comes into your head throughout the trial. You do not need to try and explain your thoughts and you should speak as often as you feel comfortable in doing so". For familiarisation, participants were also asked to TA during the warm-up. The researcher positioned themselves out of sight during the $T$ to minimise intrusion and proximity to the participant in order to reduce self-consciousness for verbalisations. Visible prompts were positioned on the handlebars as a reminder.

\section{Eye Tracking}

Participants were fitted with the eye tracking glasses, which were individually calibrated prior to completing the TT warm-up. The accuracy of which was further checked at locations on the simulation screen post-warm-up. The glasses were worn during the warm-up for familiarisation and to allow for adjustments for fit.

\section{Data Processing and Analysis}

All analyses were conducted using SPSS (Version 25) and statistical significance was accepted as $p<$ .05. Tests for normality were conducted on all data and appropriate parametric and non-parametric 
Expertise differences in cognitions, gaze behaviour and cycling performance

272

273

274

275

276

277 statistical tests subsequently used. Partial eta squared $\left(n p^{2}\right)$, Cohen's $d$ and $r$ values are reported as effect sizes.

\section{Performance Data}

Between-group differences in TT performance times were explored using an independent-samples ttest. For percentage of average speed, power, speed, heart rate and cadence, 2 (group) x 4 (distance quartile) mixed ANOVAs were conducted to explore differences between the trained and untrained groups and changes across the TT. Where significant main or interaction effects were found, Bonferonni-adjusted post hoc analyses were used to assess pairwise comparisons. Greenhouse Geisser corrections were applied where sphericity was violated.

\section{Think Aloud Data}

The TA data for each participant were transcribed verbatim and time-stamped so that verbalisations could be separated by distance quartile. A content analysis approach was taken and the data were then analysed using both inductive and deductive content analyses. Where a deductive approach was taken, the metacognitive framework previously used by Whitehead et al. (2018) and originally adopted from Brick, Maclntrye, and Campbell (2014) was used. Using this framework, verbalisations were first coded into broader primary themes (i.e., Internal Sensory Monitoring, Active SelfRegulation, Outward Monitoring and Distraction) and then further coded into more descriptive secondary themes (see Table 1). The number of themes were also grouped by distance quartile of the $\Pi$, for both the primary and secondary themes. Throughout this coding process, the researcher allowed for further inductive themes to be generated.

In-keeping with previous research that has used TA (e.g. Arsal, Eccles \& Ericsson, 2016; Whitehead et al., 2018), a post-positivist epistemology informed this study. Therefore, as recommended by MacPhail, Khoza, Abler and Ranganathan (2016), inter-rater reliability was conducted. Following the initial analysis of data using the coding framework (Table 1), a second author then analysed a $10 \%$ 
Expertise differences in cognitions, gaze behaviour and cycling performance

sample using the same framework to guide this process. Both authors compared the number and types of codes assigned to each verbalisation within the sample transcripts. Where there was a disagreement in how a verbalisation had been coded, this was marked down as a value of 1 . The total number of disagreements was summed and a percentage of total disagreements vs agreements was calculated. Within the sample, an inter-rater reliability of $86 \%$ was found. Authors engaged in discussion around the $14 \%$ disagreement and agreements were made.

To explore between-group differences in the number of verbalisations for primary and secondary themes, a series of Mann-Whitney U Tests were conducted. To explore changes in the number of verbalisations over distance quartile, Friedman's repeated-measures tests were used, followed by Wilcoxon Signed Ranks tests as post hoc analyses where significant differences were found.

\section{Eye Tracking Data}

Recorded gaze behaviour data was exported from the SMI glasses to the SMI BeGaze (Version 3.7) software to determine Areas of Interest (AOI) and subsequently map the frame-by-frame data for the TT duration. Eye-tracking data was screened for recording artefacts, eye-blinks, and missing data. Total dwell time and gaze frequency were calculated for each of the seven AOI (Time Elapsed, Power, Heart Rate, Cadence, Distance Covered, Speed, and Course Scenery). These AOIs are distributed across the bottom of the cycling simulation screen. Fixations were analysed per AOI using the BeGaze software set to "High Speed" (saccade velocity threshold set to $40^{\circ}$ a second, and fixation duration threshold as $100 \mathrm{~ms})$. Overt allocation of visual attention was defined as the relative distribution of visual dwell time ('sum of the duration of fixations and saccades within AOI', SMI 2012) and gaze frequency ('fixation count within $\mathrm{AOI}^{\prime}$ ) across each area of interest. Dwell time percentage at each AOI was calculated by dividing dwell time by the duration of the TT quartile. Dwell time was analysed for each quartile of the $T T$ and visual dwell time outside AOI's was excluded from the gaze analysis. Gaze frequency was captured for each $\mathrm{AOI}$ as further indication of the relative importance of the source of information. Gaze metrics were analysed using 2 (group) x 7 (information source) $x 4$ (distance 
Expertise differences in cognitions, gaze behaviour and cycling performance

321

322

323

324

325

326

327

328

329

330

331

332

quartile) repeated-measures ANOVAs, with group as a between-subject factor. One-way ANOVAs further explored AOI gaze behaviour differences within each TT quartile. Significant main effects were assessed with Bonferroni-adjusted post hoc comparisons.

\section{Results}

\section{Performance Data}

The trained group performed the TT in a significantly faster time than the untrained group (MD = 5.96 $\min , t(16)=4.97, p<.001, d=2.36$ ) (Table 2). A significant group main effect for speed indicated that the trained group were significantly faster than the untrained group $\left(F(1,11)=19.30, p<.001, n p^{2}=\right.$ 0.64). There was no significant main effect for quartile $\left(F(3,11)=2.03, p=.13, \eta p^{2}=.12\right)$ or group by quartile interaction $\left(F(3,11)=0.53, p=.67, n p^{2}=.01\right)$. Power was significantly higher in the trained group $\left(F(1,11)=19.03, p<.001, n p^{2}=.63\right)$ and there was a significant main effect for quartile $(F(3,11)$ $\left.=3.49, p=.026, \eta p^{2}=0.24\right)$, however no differences were found following post-hoc comparisons. The interaction effect was not significant $\left(F(3,11)=0.23, p=.87, \eta p^{2}=.46\right)$. For cadence, there was a significant main effect for group $\left(F(1,11)=7.39, p=.020, n p^{2}=.40\right)$, indicating that the trained group had a higher cadence than the untrained group. There was no main effect for quartile $(F(1.78,19.55)$ $\left.=1.39, p=.27, n p^{2}=.11\right)$ or interaction effect $\left(F(3,11)=1.22, p=.31, \eta p^{2}=.10\right)$. There were no significant main or interaction effects for heart rate or percentage of average speed (Figure 1).

\section{Think Aloud Data}

The total number of verbalisations significantly differed between the trained (Mean Rank $=4.17$ ) and untrained groups (Mean Rank $=9.43 ; \cup(13)=4.00, p=.015, r=.67$ ). Therefore, in order to allow for more accurate, relative comparisons between groups, the absolute number of verbalisations was transformed into percentage data and used in all subsequent analysis. Overall, Active Self-Regulation was the most frequently verbalised theme for the trained group, accounting for $40 \%$ of their total 
Expertise differences in cognitions, gaze behaviour and cycling performance

thoughts. On the other hand, Outward Monitoring was most frequently verbalised by the untrained group (37\%) (Table 3).

Between-group comparisons of secondary themes for the whole trial identified that the trained group verbalised significantly more Internal Self-Monitoring thoughts than the untrained group (M Ranks = 9.83 and $4.57, \mathrm{U}=4.00, p=.015, r=.67)$. The trained group verbalised more Internal SensoryMonitoring thoughts than the untrained group at quartile 3 (M Ranks 9.83 and 4.75, $\mathrm{U}=4.00, p=.015$, $r=.70)$. The untrained group verbalised significantly more Outward Monitoring thoughts at quartile 1 ( $\mathrm{M}$ Ranks $=9.07$ and $4.58, \mathrm{U}=6.50, p=.038, \mathrm{r}=.60$ ) and quartile 3 ( $\mathrm{M}$ Ranks $=9.00$ and $4.67, \mathrm{U}=7.00$, $p=.045, r=.58)$. Additionally, the untrained group verbalised more Distraction thoughts at quartile 4 ( $\mathrm{M}$ Ranks $=9.29$ and $4.33, \mathrm{U}=5.00, p=.02, \mathrm{r}=.67$ ).

For primary themes across the whole trial, the trained group verbalised more thoughts relating to Power (M Ranks $=9.67$ and 4.71, $\mathrm{U}=5.00, p=.022, r=.63$ ), whereas the untrained group were found to verbalise more thoughts relating to the Course Scenery ( $\mathrm{M}$ Ranks $=9.43$ and 4.17, $\mathrm{U}=4.00, p=.015$, $r=.67)$ and Time (M Ranks $=9.0$ and 4.67, $U=7.00, p=.045, r=.56$ ). No other significant differences in primary themes were found between the trained and untrained groups. Significant between-group differences across distance quartile are presented in Table 4 for primary themes.

Within-group differences across quartile were explored and a main effect was found for Outward Monitoring $\left(x^{2}(3)=14.46, p=.002\right)$ for the trained group. Post hoc analyses identified that they verbalised more in quartile 4 compared to $1(Z=-2.03, p=.043, r=.83)$. No significant differences were found across distance quartile for the other themes nor for the untrained group. For the primary themes, within-group analyses of cognitions across distance quartile demonstrated significant main effects for Distance $\left(x^{2}(3)=15.11 p=.002\right)$ and Power $\left(x^{2}(3)=8.53, p=.036\right)$ for the trained group. Post hoc analyses, as presented in Table 5, demonstrated that verbalisations of Distance increased throughout the trial and thoughts of Power were highest in quartile 2 and lowest in the final distance quartile. For the untrained group, significant main effects were found for the themes Distance $\left(x^{2}(3)\right.$ 
Expertise differences in cognitions, gaze behaviour and cycling performance

369

370

371

372

373

374

375

376

377

378

379

380

381

382

383

384

385

386

387

388

389

390

391

392

393

$=8.66, p=.034)$ and Motivation $\left(x^{2}(3)=13.35, p=.004\right)$. Post hoc analyses showed that verbalisations of both of these themes increased across the trial. There was also a main effect for Pace for the untrained group $\left(x^{2}(3)=8.61, p=.035\right)$, with verbalisations decreasing throughout the trial.

\section{Analysis of Percentage Dwell Time}

Means and standard deviations for percentage dwell time are presented in Table 6. A 2 (group) $x 7$ (information source) $\times 4$ (distance quartile) repeated-measures ANOVA assessed percentage dwell time within each information source across each quartile. A main effect of quartile indicated a significant decrease in dwell time within information sources as the trial progressed $(F(2.05,22.50)=$ 21.76, $p<.001, \eta p^{2}=.66$ ). Post hoc tests revealed that dwell time across all information sources was significantly lower at quartile $4(4.04 \pm 0.53)$ than at quartiles $1(7.34 \pm 0.73, p<.001), 2(6.95 \pm 0.80$, $p<.001)$, and $3(6.23 \pm 0.81, p=.007)$. Quartile 3 was also lower than quartile $2(p=.005)$, however quartile 1 was not different from quartiles $2(p=1.00)$ or $3(p=.30)$ (Figure 2$)$.

A main effect of information source $\left(F(1.44,15.87)=16.08, p<.001, \eta p^{2}=.59\right)$, indicated a significant difference in dwell time across information sources, however this was qualified by an interaction with quartile $\left(F(2.81,30.87)=518.03, p=.025, n p^{2}=.25\right)$, but not with group. There was no significant between-subjects main effect for group $\left(F(1,11)=0.24, p=.64, n p^{2}=.02\right)$ nor were any other significant interaction effects found.

At quartile 1, a one-way repeated-measures ANOVA identified a significant difference in dwell time across information sources $\left(\mathrm{F}(1.62,19.41)=11.24, p<.001, \eta \mathrm{p}^{2}=.48\right)$ (Figure 3). Post hoc tests revealed that dwell time for Course Scenery $(26.08 \% \pm 4.65)$ was significantly higher than Cadence $(3.11 \% \pm 0.74, p=.010)$, Heart Rate $(3.53 \% \pm 0.76, p=.006)$, Distance $(3.67 \% \pm 1.00, p=.010)$, Speed $(1.79 \% \pm 0.89, p=.009)$ and Time $(0.73 \% \pm 0.26, p=.003)$, but not significantly higher than Power $(12.18 \% \pm 4.79, p=1.0)$. All other comparisons were not different. At quartile 2 , a one-way repeatedmeasures ANOVA identified a significant difference in dwell time across information sources ( $F(1.58$, $\left.19.01)=13.44, p<.001, n p^{2}=.53\right)$. Post hoc tests revealed that dwell time for Course Scenery $(27.94 \%$ 
Expertise differences in cognitions, gaze behaviour and cycling performance

394

395

396

397

398

399

400

401

402

403

404

405

406

407

408

409

410

411

412

413

414

415

416

417

$\pm 5.77)$ was significantly higher than Cadence $(1.90 \% \pm 0.54, p=.014)$, Heart Rate $(2.66 \% \pm 0.71, p=$ $.022)$, Distance $(3.95 \% \pm 0.94, p=.042)$, Speed $(1.81 \% \pm 0.96, p=.026)$ and Time $(0.82 \% \pm 0.30, p=$ $.011)$, but not significantly higher than Power $(9.22 \% \pm 3.20, p=.50)$. All other comparisons were not different. At quartile 3, a one-way repeated-measures ANOVA identified a significant difference in dwell time across information sources $\left(F(1.44,17.29)=12.45, p=.001, \eta p^{2}=.51\right)$. Post hoc tests revealed that dwell time for Screen $(23.40 \% \pm 5.18)$ was significantly higher than Cadence $(1.86 \% \pm$ $0.72, p=.031)$, Heart Rate $(2.97 \% \pm 0.79, p=.044)$, Speed $(1.45 \% \pm 0.58, p=.034)$ and Time $(0.80 \% \pm$ $0.30, p=.019)$, but not significantly higher than Power $(7.36 \% \pm 2.08, p=.453)$ and Distance $(5.58 \% \pm$ $1.40, p=.154)$. All other comparisons were not different. At quartile 4 , a one-way repeated-measures ANOVA identified a significant difference in dwell time across information sources $(F(1.44,17.32)=$ $\left.10.59, p=.002, \eta p^{2}=.47\right)$. Post hoc tests revealed that dwell time for Screen $(14.41 \% \pm 3.32)$ was significantly higher than Cadence $(0.94 \% \pm 0.28, p=.027)$ and Time $(0.73 \% \pm 0.28, p=.029)$, but not significantly higher than Power $(3.85 \% \pm 1.22, p=.040)$, Heart Rate $(2.29 \% \pm 0.65, p=.091)$, Distance $(5.01 \% \pm 1.33, p=.75)$ and Speed $(1.17 \% \pm 0.42, p=.055)$. Percentage dwell time for Distance $(5.02 \%$ $\pm 1.33)$ was significantly higher than Speed $(1.17 \% \pm 0.42, p=.031)$. All other comparisons were not different.

\section{Analysis of Gaze Frequency}

Gaze frequency data are presented in Table 7. A 2 (group) $\times 7$ (information source) $\times 4$ (distance quartile) repeated-measures ANOVA assessed gaze frequency within each information source across each quartile. A main effect of quartile indicated a significant decrease in gaze frequency within information sources as the trial progressed $\left(F(2.03,22.33)=22.02, p<.001, \eta p^{2}=.67\right)$ (Figure 4). Post hoc tests revealed that gaze frequency across all information sources was significantly lower in quartile $4(75.70 \pm 9.12)$ than in quartiles $1(122.97 \pm 8.63, p<.001), 2(113.72 \pm 9.35, p<.001)$ and 3 $(111.89 \pm 11.86, p=.004)$. 
Expertise differences in cognitions, gaze behaviour and cycling performance

418 A main effect of information source indicated a significant difference in gaze frequency across AOI

$419\left(\mathrm{~F}(1.45,15.92)=15.20, p<.001, \eta p^{2}=.58\right)$ (Figure 5). Post hoc tests revealed that gaze frequency for 420 Course Scenery (398.17 \pm 73.32$)$ was significantly higher than Cadence (35.09 $\pm 7.28, p=.009)$, Heart Rate $(53.80 \pm 11.52, p=.018)$, Speed $(30.65 \pm 15.43, p=.020)$ and Time $(15.35 \pm 5.32, p=.004)$, but not Power $(129.00 \pm 39.14, p=.41)$ or Distance $(80.43 \pm 17.88, p=.058)$. Gaze frequency for Distance was significantly higher than Time $(p=.04)$. The information source $x$ quartile interaction $(F(2.63$, $\left.28.99)=2.79, p=.07, n p^{2}=.20\right)$, information source $x$ group $\left(F(1.45,15.92)=1.00, p=.37, n p^{2}=.08\right)$, and group $x$ quartile $\left(F(3,33)=.76, p=.53, \eta p^{2}=.06\right)$ interactions were not significant.

\section{Discussion}

The aim of this study was to investigate expert-novice differences in information seeking behaviour, cognitions and performance during a cycling TT. Secondly, it was aimed to identify how these behaviours and cognitions may change over the course of the time trial. Despite the trained group expectedly performing faster and at a higher mean power output and cadence than the untrained group, no significant differences in pacing strategy (i.e. power output distribution) or heart rate were observed. There was a trend for both groups to finish the trial at a faster pace, supporting previous findings of an endspurt in the final stages of an exercise bout (Taylor \& Smith, 2013). Overall, dwell time was highest for the course scenery and power output but no significant differences were found in information seeking behaviour between the trained and untrained groups, which did not support the hypothesis. For the verbalisations, there were differences in the cognitive strategies used by trained and untrained participants which supported the study predictions. Trained cyclists' cognitions were more performance-relevant during the trial (i.e. thoughts of power output), whereas the untrained group were more focused on task completion and irrelevant, distraction cues (i.e. thoughts of distance, time and the course scenery). Both groups talked more about distance covered/remaining 
Expertise differences in cognitions, gaze behaviour and cycling performance

Overall, the course scenery was the most commonly viewed information source by both groups, but the untrained group verbalised significantly more about the course than the trained group. This suggests that whilst both groups may be attending to the same visual cues, only the untrained group were using this as a dissociative strategy. Similar findings have been reported in cycling (Whitehead et al., 2018) and running, where inexperienced runners were found to report distractive thoughts including the scenery, route, other people or conversing (Brick et al., 2018). Interestingly, the untrained group verbalised a significantly greater amount of thoughts than the trained group over the duration of the trial which suggests that thinking aloud in itself may have been a distraction strategy, similar to conversing in runners (Brick et al., 2018). Conversely, power output feedback was the trained group's secondary visual source, and this was their second most verbalised theme, which was significantly higher than the untrained group. This supports evidence that trained athletes use taskrelevant cognitive strategies (Brick et al., 2015; Whitehead et al., 2017) and attend to visual domainspecific, performance feedback (Boya et al., 2017). Brick et al. (2018) found that no recreational runners used active self-regulatory strategies other than pace or tactics supporting the betweengroup difference found in the current study. It could be argued that less experienced athletes do not need the knowledge of more intricate, task-specific strategies such as the monitoring of performance data, e.g., power output or speed, or that perhaps they are less familiar with how to use it to aid performance. On the other hand, more skilled athletes use domain-specific strategies, developed through experience, to monitor and optimise pace (Nietfeld, 2003). This supports classic attentional focus research where elite runners use associative strategies and internal feedback to optimise their pace (Morgan \& Pollock, 1977). Attending to power output feedback and verbalising power-related thoughts demonstrates that experienced cyclists were continually monitoring performance to stay task-focused, supporting the provision that expert selectively allocate attentional resources to task relevant stimuli (Brams et al., 2019). untrained groups and was also the untrained group's second most viewed information source. For 
Expertise differences in cognitions, gaze behaviour and cycling performance

untrained athletes, using distance or time as a chunking strategy has been perceived to be a beneficial self-regulatory strategy (Brick et al., 2018) and is associated with increased self-efficacy and task persistence (Stock \& Cervone, 1990). This strategy allows individuals to focus on, and attain, proximal subgoals during endurance exercise. Trained athletes' primary theme of distance combined with attending to and verbalisations relating to power output, highlights more dynamic pacing judgements. Without experience-primed understanding of performance data, the untrained group may have combined distance and time feedback (their second most verbalised information source) to inform their pacing decisions.

Another aim was to explore temporal characteristics of cognitions and information seeking behaviour during the time trial. In the first half of the trial, visual course simulation and power output data dominated attentional allocation. The trained group also verbalised more about power output in the first quarter of the $T$. These visual sources were attended to significantly more than all other sources up until $12 \mathrm{~km}$. Mestre, Maino, Dagonneau and Mercier (2011) also found that exercisers typically spent less time watching virtual-scenery video feedback as a cycling task progressed. Overall dwell time and gaze frequency significantly decreased in the final $4 \mathrm{~km}$ of the trial alongside a slight drop in the total number of verbalisations. This indicates that participants utilised more external feedback sources at the start of the trial where task uncertainty is at its peak and then were potentially more internally focused in the final quartile. The trained group's initial focus on power output data also suggests that this domain-specific cognitive strategy may be used more prominently in the monitoring and control of their initial pace and cognitions (Brick et al., 2018).

The untrained group, on the other hand, verbalised more about distance (Outward Monitoring) and the course in the first $4 \mathrm{~km}$. Their verbalisations of pace were also higher in the first half of their trial. Distance and pace verbalisations were associated with a relatively fast start and resultant unpleasant physical sensations (Brick et al., 2018). A trend for their pace to drop in the third quartile illustrates the decision that their initial pace was not sustainable, and a conscious adjustment was needed to 
Expertise differences in cognitions, gaze behaviour and cycling performance

ensure successful task completion (Edwards \& Polman, 2013; 2012), a trend that is not uncommon in less experienced athletes (Deaner, Carter, Joyner,, \& Hunter, 2015). In the third quartile, the trained group verbalised significantly more Internal Sensory Monitoring cognitions (i.e. heart rate and pain) whereas the untrained group verbalised significantly more Outward Monitoring cognitions (i.e. time), and about the course and hydration. Untrained athletes appear to adopt a more dissociative strategy, yet this is typically associated with a poorer performance (LaCaille, Masters, \& Heath, 2004; Morgan \& Pollock, 1977) which is reflected by the drop in pace. Whilst the trained group verbalised more internally-driven thoughts at this stage, they maintained a relatively even pace, suggesting that their attention to, and interpretation of, these sensory cues may be different to that of the less experienced group. This would support the conscious awareness brain regulation model of exercise regulation (Edwards \& Polman, 2012).

Both groups increased the number of verbalisations relating to distance in the final $4 \mathrm{~km}$ and this was also associated with an increase in dwell time on distance feedback for both groups. Therefore, it appears that cognitions and information seeking became more selective and driven by end-point knowledge, i.e. distance, and that cognitive strategies change towards the end of the exercise bout. Rather than thinking about task-relevant factors to monitor and control pace, with fewer pacing decisions left to make and less uncertainty as the endpoint approached (St Clair Gibson et al., 2006), the trained group shifted their focus from power output feedback to covering the remaining distance in the fastest time possible. On the other hand, the untrained group paid less attention to distracting information (i.e. Course Scenery) and similarly appeared focused on task completion, supporting the active self-regulatory approaches observed in other research (e.g., Brick et al., 2018).

The untrained group also verbalised significantly more motivational cognitions in the final quartile compared to the preceding quartiles. The use of motivational self-talk has been shown to be an adaptive strategy because it reduces perceptions of effort and improves performance (Blanchfield, Hardy, deMorree, Staino, \& Macrora, 2014; Weinberg, Miller, \& Horn, 2012). Recreational runners in 
Expertise differences in cognitions, gaze behaviour and cycling performance

518

519

520

521

522

523

524

525

526

527

528

529

530

531

532

534

535

536

537

538

539

540

541

Brick et al.'s (2018) study reported metacognitive feelings about knowing when to apply a cognitive strategy and that this is dependent on factors such task duration and physical sensations. Using motivational self-talk when the endpoint is approaching and physical exertion at its highest, is no surprise based on these previous findings. For both groups, this cognitive strategy was associated with an observed increase in pace supporting its use to enhance performance (Blanchfield et al., 2014; Weinberg et al., 2012) and that pacing at higher levels of exertion is more likely to be regulated by conscious processes (Edwards \& Polman, 2012).

Study 2: Investigation of the influence of a competitor on performance and cognitions in trained and untrained cyclists.

In addition to the visual cues explored above, many endurance events also involve other athletes, including teammates or competitors. With most previous research in pacing exploring the influence of a competitor on the performance of trained athletes (see Konings \& Hettinga, 2018), whether training status and experience is an important factor in an individual's response to competitive situations requires investigation. Therefore, this study aimed to explore the effect of a virtual competitor on cognitions and cycling $\Pi$ performance with trained cyclists and physically active individuals. It was hypothesised that both groups would perform faster when against a competitor but that cognitions would differ between the groups.

\section{Methods}

\section{Design}

A 2 (group) $\times 2$ (trial) $\times 4$ (distance quartile) design was used to compare differences in performance and cognitions between trained and untrained participants. Participants performed three $16.1 \mathrm{~km}$ cycling TTs on separate visits, 3-6 days apart; an initial baseline TT, an alone TT (ALONE) and a TT against a virtual competitor (COMP). The baseline TT was performed for familiarisation purposes and to record the participants' performance for use in the subsequent COMP trial and was therefore not 
Expertise differences in cognitions, gaze behaviour and cycling performance

542

included in the analyses. The ALONE and COMP trials were performed in a counterbalanced order. The TA protocol was used to record verbalisations throughout each trial and performance time (min:s), speed $\left(\mathrm{km} \cdot \mathrm{hr}-{ }^{1}\right)$, power output $(\mathrm{W})$, heart rate (beats. $\mathrm{min}^{-1}$ ) and cadence (revs. $\mathrm{min}^{-1}$ ) were continuously recorded. Data were analysed across distance quartile to explore changes over time.

\section{Participants}

Eight trained male cyclists $(M$ age $=48.5 \pm 14.6$ years; $M$ height $=174.3 \pm 5.5 \mathrm{~cm} ; M$ body mass $=68.4$ $\pm 4.6 \mathrm{~kg}$ ) and ten untrained, physically active males ( $M$ age $=34.9 \pm 5.9$ years; $M$ height $=177.3 \pm 4.0$ $\mathrm{cm} ; M$ body mass $=85.4 \pm 13.7 \mathrm{~kg}$ ) participated in the study. The inclusion criteria for each group as stated in Study 1 were replicated in this study.

\section{Materials}

The Velotron electromagnetically-braked cycle ergometer and RacerMate software, described in Study 1 , were used for all three trials and testing was based in a laboratory. Participants wore a Polar heart rate monitor in each trial and an Olympus Dictaphone was used to capture the in-event thoughts that were verbalised. The Dictaphone microphone was fitted to the participants' collar to ensure clarity of sound with the wire placed inside the shirt and connected to the recording device placed in the back pocket of the cycling jersey.

\section{Procedure}

In addition to control measures outlined in Study 1, participants were also asked to replicate, as much as was practically possible, their eating, drinking, sleeping and exercise behaviour in the 24 hours preceding each trial. The cycle ergometer was adjusted to suit the participant's stature on the first visit and then replicated exactly in subsequent trials. During the BL and ALONE trials, participants performed by themselves with just the performance feedback displayed on the screen. During the COMP trial, a simulated virtual avatar was projected onto the screen and participants were instructed that this avatar represented a competitor's performance that was comparable to their BL effort. The 
Expertise differences in cognitions, gaze behaviour and cycling performance

566

567

568

569

570

571

572

573

574

575

576

577

578

579

580

581

582

583

584

585

586

587

588

589

avatar was in fact an exact replication of the speed profile of the participants' BL trial. Water only was consumed during the exercise, without excessive variation between trials. A standing fan was offered to participants and settings again replicated in each trial.

\section{Data Analysis}

To analyse differences in performance times, a mixed 2 (group) $\times 2$ (trial) ANOVA was performed. Mixed ANOVAs (2 (group) $\times 2$ (trial) $\times 4$ (distance quartile)) were conducted to analyse differences in power output, pace (percentage of average speed), heart rate and cadence. Where significant main or interaction effects were found, Bonferroni-adjusted post hoc comparisons were conducted.

Data for TA were analysed as described in Study 1. To compare differences between the trained and untrained groups in both the ALONE and COMP trials, between-group comparisons of secondary and primary themes were made using Mann-Whitney U tests. Wilcoxon Signed Rank tests were then used to compare differences between the ALONE and COMP trials for both the trained and untrained groups. To compare differences in primary and secondary verbalisations across the duration of the trials, Friedman's tests were used to analyse within-group distance quartile changes across both the ALONE and COMP trials. Significant quartile effects were followed up with Wilcoxon Ranks post hoc comparisons.

\section{Results}

\section{Performance Data}

Means and standard deviations for performance data are presented in Table 8. For performance time, a significant group main effect $\left(\mathrm{F}(1,16)=30.32, p<.001, \mathrm{np}^{2}=.66\right)$ was found demonstrating that the trained group performed both TTs in a significantly faster time than the untrained group. There was a non-significant main effect for trial $\left(F(1,16)=0.01, p=.94, n p^{2}=.001\right)$ and for the trial by group interaction $\left(F(1,16)=1.23, p=.28, n p^{2}=.07\right)$. For power output, a significant main effect for group was found $\left(F(1,16)=38.73, p<.001, \eta p^{2}=.71\right)$, where power was found to be significantly higher in the 
Expertise differences in cognitions, gaze behaviour and cycling performance

590

591

592

593

594

595

596

597

598

599

600

601

602

603

604

605

606

607

608

609

610

611

612

613

614

trained group. A significant main effect for quartile was also found $\left(F(1,16)=9.15, p=.001, \eta p^{2}=.36\right)$, with post hoc analyses demonstrating that power was higher in quartile 4 than both quartiles 2 (MD $=-13.96 \mathrm{~W}, p=.045, \mathrm{Cl}-27.71,-0.21)$ and $3(\mathrm{MD}=-17.01 \mathrm{~W}, p=.002, \mathrm{Cl}=-28.01,-6.02)$. A significant group by quartile interaction effect demonstrated that the trained group performed at a higher power output than the untrained group in all quartiles $(p<.001)$.

For pace, represented as a percentage of average speed, a significant main effect for quartile was found $\left(F(2.13,34.14)=5.32, p=.009, n p^{2}=.25\right)$, where pace was significantly slower in quartile 3 than $4(\mathrm{MD}=-2.59 \%, p=.007, \mathrm{Cl}-4.57,-0.60)$; indicative of an endspurt, regardless of condition or group (Figure 6). Post-hoc analyses following a significant interaction between quartile and group for pace $\left(F(3,35)=4.00, p=.013, n p^{2}=.20\right)$ showed differences between groups in quartiles $2(M D=1.67, p=$ $.026, \mathrm{Cl}=0.23,3.11)$ and $3(\mathrm{MD}=1.67, p=.031, \mathrm{Cl}=0.17,3.18)$. Therefore, regardless of condition, trained and untrained participants pace themselves differently during the middle portion of the trial, with trained participants producing a higher percentage of average speed than the untrained group at these two quartiles. A significant group main effect for cadence highlighted that the trained group had a higher cadence than the untrained group $\left(F(1,16)=13.28, p=.002, n p^{2}=.45\right)$. There was a significant interaction effect for heart rate between trial and group $\left(\mathrm{F}(1,16)=4.56, p=.048, n p^{2}=.22\right)$ however no significant post hoc differences were found. No other significant main or interaction effects were found.

\section{Think Aloud Data}

A Mann-Whitney $U$ test revealed that the total number of verbalisations did not significantly differ between the trained (Mean Rank $=7.13$ ) and untrained groups (Mean Rank $=10.67, \mathrm{U}(16)=21.00, p$ $=.15, r=.35)$. As presented in Table 9, Active Self-Regulation was the most commonly verbalised theme for both groups in both trials, with Distraction being the least verbalised theme.

No significant differences were found for any secondary theme in the ALONE trial $(p>.05)$. In the COMP trial, the trained group were found to verbalise significantly more Active Self-Regulation 
Expertise differences in cognitions, gaze behaviour and cycling performance

615

616

617

618

619

620

621

622

623

624

625

626

627

628

629

630

631

632

633

634

635

636

637

638

639

thoughts than the untrained group (Mean Ranks $=10.13$ and 5.57, $\mathrm{U}=11.00, p=.049, \mathrm{r}=.51$ ). No significant differences in secondary themes were found between the ALONE and COMP trials for either group $(p>.05)$. For primary themes, the untrained group verbalised significantly more thoughts of Pace, Technique and Time in the ALONE trial than the trained group (Table 10). In the COMP trial, the trained group verbalised more thoughts of Power than the untrained group, whereas the untrained group verbalised more thoughts of Pace. No significant differences were found between trials for any primary theme in either group $(p>.05)$.

For secondary themes, a significant change over quartile was found for Outward Monitoring thoughts in the ALONE trial for the trained group $\left(x^{2}(3)=11.21, p=.011\right)$. Significantly more verbalisations were found in quartile 3 than quartiles $1(Z=2.20, p=.028, r=.78)$ and $2(Z=-1.99, p=.046, r=.70)$, as well as more verbalisations in quartile 4 than quartiles $1(Z=2.20, p=.028, r=.78)$ and $2(Z=-1.99, p$ $=.046, r=.70)$.

For primary themes, significant quartile main effects for Motivation were found for both the trained $\left(x^{2}(3)=8.51, p=.037\right)$ and untrained groups $\left(x^{2}(3)=10.81, p=.013\right)$ in the ALONE trial. Post hoc analyses demonstrated that both groups verbalised significantly more Motivation thoughts in the final quartile (Table 11). The same pattern was also found in the COMP trial for the trained group $\left(x^{2}(3)=\right.$ $10.90, p=.012)$. Significant quartile main effects were found for cadence for the trained group in both the ALONE $\left(x^{2}(3)=10.85, p=.013\right)$ and COMP trials $\left(x^{2}(3)=9.73, p=.021\right)$ but post hoc analyses were not significant. In the COMP trial, a quartile main effect for Pace $\left(\mathrm{x}^{2}(3)=8.18, p=.042\right)$ demonstrated that the untrained group verbalised the most amount of Pace thoughts in quartile 2. Lastly, a significant effect for Distance $\left(x^{2}(3)=12.11, p=.007\right)$ showed that the trained group verbalised significantly more in the second half of the ALONE trial.

\section{Discussion}

This study aimed to explore the effect of a virtual competitor on cognitions and cycling TT performance between trained and untrained individuals. The trained group performed the trials in a faster time 
Expertise differences in cognitions, gaze behaviour and cycling performance

640

than the untrained group, maintaining a higher average power output. Whilst both groups produced endspurts, demonstrated by a faster final quartile, the trained group performed at a faster pace (i.e., a higher percentage of average speed) in the middle section of the trials compared to the untrained group which is indicative of a more even pacing profile. Contrary to our prediction, no differences in performance were found for either group between the ALONE and COMP trials suggesting that the presence of a competitor did not influence their pacing strategy.

The finding that pace was unaffected by the presence of a competitor contradicts previous evidence that endurance performance is improved when trained athletes perform against a virtual (Williams et al., 2014) or actual competitor (Corbett et al., 2012). A reason for this could be that additional performance data (speed, distance, time, power, heart rate, cadence) was presented as visual feedback throughout all trials in the present study, creating a more complex performance environment (Hettinga et al., 2017a). Participants' decisions for action were therefore informed by the availability of internal factors (fatigue, pain), external performance feedback (visual performance data) and the social environment (virtual competitor behaviour). Having access to multiple feedback sources, and with the suggestion that only certain affordances can survive (Hettinga et al., 2017a), it appears that participants may have prioritised performance feedback over the behaviour of the virtual competitor. This is supported by the cognitions in the COMP trial, where $46 \%$ of verbalisations referred to visual performance data available (i.e., speed, distance, power, time, heart rate and cadence) whereas only $11 \%$ of verbalisations related to the virtual competitor, suggesting that the competitor was less useful for regulating performance.

An alternative explanation for the absence of a change in performance during competitive TTs could be the inability of the competitor to provide a substantial increase in the athletes' motivation. Other studies have also found that external factors including a competitor (Bath et al., 2012) or monetary rewards (Hulleman, de Koning, Hettinga, \& Foster, 2007) do not affect performance. Bath et al. (2012) concluded that whilst the competitor was intended as an external motivator, it was not sufficient to 
Expertise differences in cognitions, gaze behaviour and cycling performance

665

666

667

668

669

670

671

672

674

675

676

677

678

679

680

681

682

684

685

686

687

688

689

increase work rate to experience a greater level of physiological discomfort. Similarly, a study by Hibbert, Billaut, Varley and Polman (2018) found that $5 \mathrm{~km}$ TT performance was not improved when competing against three actual cyclists. Although, athletes with an ego orientation were vulnerable to decreased performance. Of interest, despite a minimal change in work rate in the competitor TT compared to the alone $\pi$, trained participants' heart rate values were on average $\sim 20 \mathrm{bpm}$ higher when performing against a competitor. Whilst not improving performance, the competitive environment instilled an arousal-induced increase in heart rate, illustrating that trained athletes may have been more psycho-physiologically influenced by an opponent than those less familiar with the task.

Whilst both groups verbalised different thoughts, this did not differ between the ALONE and COMP conditions. The untrained group verbalised more thoughts of Pace and Time than the trained group in the ALONE trial. The use of time elapsed and pace as cognitive calculations of current performance (see Untrained Pace quote in Table 1), could be due to inexperience of the task and their uncertainty requiring more continuous monitoring, in the absence of a robust, pre-set pacing schema (Micklewright, Papadopoulou, Swart, \& Noakes, 2010). This is further supported by the greater Pace verbalisations in the first half of the COMP trial compared to the latter stages of the trial. The presence of a competitor, whilst not influencing performance, creates a more complex performance environment and therefore untrained and unfamiliar individuals may need to more consciously attend to pacing-related affordances during the initial stages of a competitive task (Hettinga et al., 2017a).

The trained group verbalised more Active Self-Regulation and Power thoughts than the untrained group in the COMP trial. Whilst Active Self-Regulation (i.e., task-relevant, performance-focused thoughts) has been consistently found to be the most commonly verbalised theme in TA research (see Study 1; Whitehead et al., 2018), the finding that trained athletes verbalised this theme significantly more than untrained athletes is unique to the present study. Furthermore, the proportion of Active Self-Regulation thoughts increased by over $10 \%$ from the ALONE to the COMP trial in the trained 
Expertise differences in cognitions, gaze behaviour and cycling performance

690

691

692

693

694

695

696

697

698

699

700

701

702

703

704

705

706

707

708

709

710

711

712

713

714

group. This novel finding was evident in the COMP trial only suggesting that during a more competitive task, trained athletes may use more performance-driven cognitive strategies than untrained athletes. Through experience, they are better able to remain focused on their own performance (i.e. power output feedback) despite the distracting presence of a virtual competitor. Changes in cognitions across the duration of the ALONE trial were evident for the trained group, as they verbalised more Outward Monitoring thoughts driven by an increase in Distance thoughts, in the second half of this trial. Consistent with previous TA research (Study 1; Whitehead et al., 2018; 2017), this supports the argument that trained athletes use distance information to appraise their performance as they near goal attainment.

When approaching the task endpoint, both groups verbalised more Motivation thoughts in the final quartile of their ALONE trial and the trained group also verbalised more in the final quartile of the COMP trial. This supports Study 1 and previous research (Whitehead et al., 2017) in indicating a positive self-talk strategy (Blanchfield et al., 2014; Barwood, Corbett, \& Wagstaff, 2015). As a task becomes more challenging and it becomes more salient to overcome greater levels of perceived discomfort and maintain a target pace (Brick, Maclntyre, \& Campbell, 2016), there is a greater need for cognitive strategies to enable goal attainment. In the current study, this strategy aligned with both groups producing an increase in pace in the final quartile; an endspurt, that is often observed in endurance events (Lima-Silva et al., 2013).

The present study observed no effect of a competitor on pace or performance in trained or untrained populations. Cognitive appraisals indicated that performance feedback may have instead been prioritised in this complex performance environment rather than this external environmental stimulus. Differences between trained and untrained athletes also suggest that those with more experience may be better at remaining performance-focused when exposed to these external distractions. On the other hand, untrained athletes less familiar with the given task needed to make more conscious appraisals of their pace due to uncertainty and lack of an experience-primed pacing 
Expertise differences in cognitions, gaze behaviour and cycling performance

schema. It is also worth noting that the novel application of TA may have interfered with the otherwise commonly observed facilitative competitor presence effects. Finally, regardless of expertise, athletes use motivational cognitive strategies in the latter stages of the task to facilitate an increase in pace.

\section{General Discussion}

The two studies presented explored differences in cognitions and performance between trained and untrained participants in cycling time trials using novel measures. Study 1 incorporated eye tracking techniques to identify differences in visual search strategies and Study 2 explored changes in cognitions and performance when performing against a virtual competitor. As expected, trained cyclists perform all TTs in a faster time than untrained participants with no experience of cycling TTs. In Study 2, trained athletes also produced a more even pacing profile than the untrained group which has been suggested to be the optimal pacing strategy for an endurance event of this distance (Abbiss \& Laursen, 2008) and therefore supports the importance of experience in pacing success. Study 1 demonstrated that expertise did not influence overt information seeking behaviour, with no differences in gaze behaviour evident between groups. Additionally, there were no differences in pace or performance identified between groups when participants performed against a competitor in Study 2. However, differences in cognitions were found between groups in both studies, demonstrating that expertise is a factor that influences the cognitive strategies used during endurance performance.

Overall, Active Self-Regulation was the most prominent theme verbalised by both groups, consistent with previous Think Aloud research within cycling (Whitehead et al., 2018) highlighting these cognitions accounting for $40-63 \%$ of thoughts during cycling time trials. In relation to Brick et al.'s (2014) metacognitive framework, our results support metacognitive skills differing between trained and untrained participants. Specifically, trained cyclists use more Active Self-Regulatory strategies (e.g. use of power output feedback) than untrained counterparts indicating more domain-specific, performance-related focus of attention is used to monitor pace and goal attainment, consequently supporting the information-reduction hypothesis (Brams et al., 2019). On the other hand, untrained 
Expertise differences in cognitions, gaze behaviour and cycling performance

individuals verbalised more about time and distance suggesting a focus on task completion and cognitive strategies such as chunking or dissociation to better tolerate unpleasant physical sensations.

More irrelevant, distracting cognitive strategies were used by the untrained group. Given that these types of strategies are typically associated with reduced perceptions of exertion, slower pace and increased positive affect (LaCaille et al., 2004), it does not necessarily indicate that it is a poor strategy for less experienced athletes to use. Whilst the trained group were presumably aiming for the fastest possible performance, the untrained may have been satisfied with task completion and enjoyment of the task. This strategy may therefore be more beneficial as enjoyment and positive affect are associated with long-term adherence to endurance activity (Brick et al., 2018; Williams, 2008).

In both studies, the trained groups verbalised more Distance and Outward Monitoring thoughts in the latter stages of the trials, and a similar trend was demonstrated in Study 1 for the untrained group. This evidenced that both groups attend to this visual information more as the dwell time on time elapsed feedback increased in the final quartile. Consistent with previous cycling studies (Whitehead et al. 2018; Whitehead et al., 2017), this suggests that cyclists constantly appraise distance information to inform their regulatory efforts, potentially in line with prior knowledge and experience (McCormick et al., 2015).

A key finding in this and other endurance TA research is that individuals, both trained cyclists and physically active individuals, verbalise more motivational thoughts in the final stages of the trial (Whitehead et al. 2018). These motivational strategies are not uncommon within pacing literature. Brick et al. (2018) also found that endurance athletes adopt self-regulatory strategies such as motivational self-talk to counter negative thoughts. The conscious awareness of cumulative fatigue and physical discomfort may trigger the need for positive, self-encouragement as a coping strategy during these final kilometres. This cognitive strategy also coincided with an increase in pace in the final $4 \mathrm{~km}$ which supports previous evidence that this may be beneficial for performance (Blanchfield 
Expertise differences in cognitions, gaze behaviour and cycling performance

764

765

766

767

768

769

770

771

772

773

774

775

776

et al., 2014; Weinberg et al., 2012). These findings also seem to support the conscious awareness brain regulation model of pacing (Edwards \& Polman, 2013).

\section{Limitations and Future Research}

This study did not identify any expert-novice differences in gaze behaviour during cycling performance. Despite this, cognitive processes were different between experts and novices, as indicated by the TA verbalisations. When undertaking challenging visuo-motor tasks, novices often direct their attention to perceptually salient features, but may not process task-relevant information (D'Innocenzo, Gonzalez, Williams, \& Bishop, 2016). As such, it is possible that visual behaviour during cycling time trials is not sensitive to expert-novice differences, whereas meta-cognitive processes that are underpinned by the acquired information are. As noted, laboratory simulation-based investigations of athletes' perceptual expertise are limited due to differences in contextual information and the often simplified representativeness of tasks (see van der Kamp, Rivas, van Doorn, \& Savelsbergh, 2008). Future research considering more naturalistic and representative settings of endurance activity is needed. For example, researchers might consider using immersive virtual environments to examine more ecologically valid search behaviour in endurance athletes.

Both present studies included novel methodologies to further understand decision-making and pacing regulation through the use of continually presented feedback, simultaneously exploring visual search behaviours and cognitive processing. Whilst the incorporation of both eye tracking techniques and the TA protocol is a novel approach of the current study, the additional secondary task of verbalising cognitions could potentially explain the differences found when compared to previous eye-tracking research within cycling (Boya et al., 2017). As a secondary task, relying on working memory, TA may also be vulnerable to problems associated with potential impairment of executive functioning observed during exhaustive exercise as a result of competition for limited processing resources (see Schmit \& Brisswalter, 2018). Furthermore, the use of concurrent TA protocols has been associated with slowing-down effects (e.g., Krings, 2001), changes in cognitive process approaches (Jakobsen, 
Expertise differences in cognitions, gaze behaviour and cycling performance

2003), and having limited access to meta-cognitive processes (van Gog, Paas, van Merriënboer \& Witte, 2005). As such, it is possible that the act of attempting to verbalise thoughts during the $T T$ has interfered with the task. For example, through conscious promotion of regulatory efforts, attention may have been actively directed to sources of information that otherwise may not have been processed. Furthermore, in human-computer interaction research it has been noted that participants who engaged in TA protocols whilst also undergoing eye-tracking during web-page use, failed to report information regarding what they had been looking at (Cooke \& Cuddihy, 2005). Future research should therefore consider caution in using both strategies concurrently as effective indications of metacognitive processes.

\section{Conclusion}

The inclusion of both ride-alone and competitive time trials, and expert and novice groups has not previously been investigated in endurance performance research to date. Similarly, this is the first known study to utilise both eye tracking techniques and the Think Aloud protocol to investigate the link between cognitive processes and information seeking behaviour in endurance exercise.

The two studies presented in this paper demonstrate that cognitive processes differ as a function of expertise during self-paced cycling time trials. Trained cyclists were found to have more domainspecific, task-relevant thoughts whereas untrained individuals may be more focused on task completion and use more dissociative cognitive strategies. No differences in information seeking behaviour were identified between the two groups with the use of eye tracking techniques in Study 1. Furthermore, neither group significantly changed their pacing strategy or performance when performing against a virtual competitor in Study 2.

From these findings it is unclear as to whether measures of overt direction of attention in the form of eye gaze data is a relevant indicator of task-specific perceptual-cognitive expertise when compared to the underlying meta-cognitive processes highlighted by the Think Aloud data. Future research would need to explore these two data collection techniques further to enhance our knowledge in these sport 
Expertise differences in cognitions, gaze behaviour and cycling performance

814

815

816

817

818

819

820

821

822

823

824

825

826

827

828

829

830

831

832

833

834

835

specific tasks. Similarly, the creation of a complex, saturated information environment may have resulted in the competitive opponent not having the same extrinsic motivation effects as previous research has shown when withdrawing personal performance feedback from athletes during exertion. For future practical application, researchers should identify feedback source preference and selected affordances for an athlete to have optimal cognitive strategies during competition.

\section{References}

Abbiss, C. R., \& Laursen, P. B. (2008). Describing and understanding pacing strategies during athletic competition. Sports Medicine, 38, 239-239. https://doi.org/10.2165/00007256-200838030-00004.

Arsal, G., Eccles, D. W., \& Ericsson, K. A. (2016). Cognitive mediation of putting: Use of a think-aloud measure and implications for studies of golf-putting in the laboratory. Psychology of Sport and Exercise, 27(1), 18-27. http://dx.doi.org/10.1016/j. psychsport.2016.07.008.

Bahrick, H. P., Hall, L. K., \& Berger, S. A. (1996). Accuracy and distortion in memory for high school grades. Psychological Science, 7(5), 265-271. https://doi.org/10.1111/j.1467-9280.1996.tb00372.x.

Baker, J., Côté, J., \& Deakin, J. (2005). Cognitive characteristics of expert, middle of the pack, and back of the pack ultra-endurance triathletes. Psychology of Sport and Exercise, 6(5), 551-558. http://dx.doi.org/10.1016/j.psychsport.2004.04.005.

Bale, J. (2006). The place of pain in running. In S. Loland, B. Skirstad, \& I. Waddington (Eds.). Pain and injury in sport: Social and ethical analysis (pp. 65-75). Oxon: Routledge.

Barwood, M. J., Corbett, J., Wagstaff, C. R. D., McVeigh, D., \& Thelwell, R. C. (2015). Improvement of 10-km time-trial cycling with motivational self-talk compared with neutral self-talk. International Journal of Sports Physiology and Performance, 10, 166- 171. http://doi.org/10.1123/ijspp.2014-0059. 
Expertise differences in cognitions, gaze behaviour and cycling performance

836

837

838

839

840

841

842

843

844

845

846

847

848

849

850

851

852

853

854

855

856

857

858

Bath, D., Turner, L. A., Bosch, A. N., Tucker, R., Lambert, E. V., Thompson, K. G., \& St Clair Gibson, A. (2012). The effect of a second runner on pacing strategy and RPE during a running time trial. International Journal of Sports Physiology and Performance, 7(1), 26-32. https://doi.org/10.1123/ijspp.7.1.26.

Blanchfield, A. W., Hardy, J., deMorree, H. M., Staino, W., \& Marcora, S. M. (2014). Talking yourself out of exhaustion: The effects of self-talk on endurance performance. Medicine and Science in Sport and Exercise, 46(5), 998-1007. https://doi.org/10.1249/MSS.0000000000000184.

Boya, M., Foulsham, T., Hettinga, F. J., Parry, D., Williams, E. L., Jones, H. S., S. Sparks, A., Marchant, D. C., Ellison, P., Bridge, C. A., McNaughton, L. R., \& Micklewright, D. (2017). Information Acquisition Differences between Experienced and Novice Time Trial Cyclists. Medicine \& Science in Sports \& Exercise, 49(9), 1884-1898. http://doi.org/10.1249/MSS.0000000000001304.

Brams, S., Ziv, G., Levin, O., Spitz, J., Wagemans, J., Williams, A. M., \& Helsen, W. F. (2019, August 15). The Relationship Between Gaze Behavior, Expertise, and Performance: A Systematic Review. Psychological Bulletin. Advance online publication. http://dx.doi.org/10.1037/bul0000207.

Brick, N. E., Campbell, M. J., Metcalfe, R. S., Mair, J. L., \& Maclntyre, T. E. (2015). Altering pace control and pace Regulation: Attentional focus effects during running. Medicine and Science in Sports and Exercise, 48(5), 879-886. http://dx.doi.org/10.1249/ MSS.0000000000000843.

Brick, N. E., Campbell, M. J., Sheehan, R. B., Fitzpatrick, B. L., \& MacIntyre, T. E. (2018). Metacognitive processes and attentional focus in recreational endurance runners. International Journal of Sport and Exercise Psychology, 1-18. https://doi.org/10.1080/1612197X.2018.1519841.

Brick, N. E., MacIntyre, T. E., \& Campbell, M. J. (2016). Thinking and action: a cognitive perspective on self-regulation during endurance performance. Frontiers in Physiology, 7, 159. https://doi.org/10.3389/fphys.2016.00159PMID:27199774. 
Expertise differences in cognitions, gaze behaviour and cycling performance

859

860

861

862

863

864

865

866

867

868

869

870

871

872

873

874

875

876

877

878

879

880

881
Brick, N. E., Maclntyre, T. E., \& Campbell, M. J. (2014). Attentional focus in endurance activity: New paradigms and future directions. International Review of Sport and Exercise Psychology, 7(1), 106134. http://dx.doi.org/10.1080/1750984x.2014.885554.

Cisek, P. (2007). Cortical mechanisms of action selection: the affordance competition hypothesis. Philosophical Transactions of the Royal Society B: Biological Sciences, 362(1485), 1585-1599. https://doi.org/10.1098/rstb.2007.2054.

Cisek, P., \& Kalaska, J. F. (2010). Neural mechanisms for interacting with a world full of action choices. Annual Review of Neuroscience, 33, 269-298. https://doi.org/10.1146/annurev.neuro.051508.135409

Cona, G., Cavazzana, A., Paoli, A., Marcolin, G., Grainer, A., \& Bisiacchi, P. S. (2015). It's a matter of mind! Cognitive functioning predicts the athletic performance in ultra-marathon runners. PloS ONE, 10(7), e0132943. https://doi.org/10.1371/journal.pone.0132943.

Cooke, L., \& Cuddihy, E. (2005). Using eye tracking to address limitations in think-aloud protocol. In IPCC 2005. Proceedings. International Professional Communication Conference, 2005 (pp. 653-658). IEEE.

Corbett, J., Barwood, M. J., Ouzounoglou, A., Thelwell, R., \& Dicks, M. (2012). Influence of competition on performance and pacing during cycling exercise. Medicine and Science in Sports and Exercise, 44(3), 509-515. doi: 10.1249/MSS.0b013e31823378b1.

D'Innocenzo, G., Gonzalez, C.C., Williams, A. M., Bishop, D. T. (2016). Looking to Learn: The Effects of Visual Guidance on Observational Learning of the Golf Swing. PLOS ONE, 11(5), e0155442. https://doi.org/10.1371/journal.pone.0155442.

Deaner, R. O., Carter, R. E., Joyner, M. J., \& Hunter, S. K. (2015). Men are more likely than women to slow in the marathon. Medicine and Science in Sports and Exercise, 47, 607-616. doi: 10.1249/MSS. 0000000000000432. 
Expertise differences in cognitions, gaze behaviour and cycling performance

882

883

884

885

886

887

888

889

890

891

892

893

894

895

896

897

898

899

900

901

902

903

904

905

De Pauw, K., Roelands, B., Cheung, S. S., de Geus, B., Rietjens, G., \& Meeusen, R. (2013). Guidelines to classify subject groups in sport-science research. International Journal of Sports Physiology and Performance, 8(2), 111-122. http://dx.doi.org/10.1123/ijspp.8. 2.111.

Dicks, M., Button, C., \& Davids, K. (2010). Examination of gaze behaviors under in situ and video simulation task constraints reveals differences in information pickup for perception and action. Attention, Perception, \& Psychophysics, 72(3), 706-720. https://doiorg.edgehill.idm.oclc.org/10.3758/APP.72.3.706.

Edwards, A. M., \& Polman, R. C. J. (2013). Pacing and awareness: Brain regulation of physical activity. Sports Medicine, 43(11), 1057-1064. https://doi.org/10.1007/s40279-013-0091-4.

Edwards, A. M., \& Polman, R. C. J. (2012). Pacing in sport and exercise: A psychophysiological perspective. New York: Nova Science Publishers.

Ericsson, K. A., \& Kirk, E. (2001). Instructions for giving retrospective verbal reports. Unpublished manuscript. Florida State University.

Ericsson, K. A., \& Simon, H. A. (1980). Verbal reports as data. Psychological Review, 87(3), 215. http://dx.doi.org/10.1037/0033-295X.87.3.215.

Hettinga, F. J., Konings, M. J., \& Pepping, G. J. (2017a). The science of racing against opponents: affordance competition and the regulation of exercise intensity in head-to-head competition. Frontiers in Physiology, 8, 118. https://doi.org/10.3389/fphys.2017.00118.

Hettinga, F. J., Renfree, A., Pageaux, B., Jones H. S., Corbett J., Micklewright D., \& Mauger A. R. (2017b). Editorial: Regulation of Endurance Performance: New Frontiers. Frontiers in Physiology, 8, 727. https://doi.org/10.3389/fphys.2017.00727.

Hibbert, A., Billaut, F., Varley, M., \& Polman, R. C. J. (2018). Goal orientation and the presence of competitors influence cycling performance. Frontiers in Psychology: Movement Science and Sport Psychology, 9, 1212. doi: 10.3389/fpsyg.2018.01212/full. 
Expertise differences in cognitions, gaze behaviour and cycling performance

906

907

908

909

910

911

912

913

914

915

916

917

918

919

920

921

922

923

924

925

926

927

Hill, A. V., \& Long, C. N. H. (1925). Muscular exercise, lactic acid, and the supply and utilisation of oxygen. Ergebnisse der Physiologie, 24(1), 43-51. https://doi.org/10.1098/rspb.1924.0037.

Hulleman, M., de Koning, J. J., Hettinga, F. J., \& Foster, C. (2007). The effect of extrinsic motivation on cycle time trial performance. Medicine and Science in Sports and Exercise, 39(4), 709-715. doi: $10.1249 /$ mss.0b013e31802eff36

Jakobsen, A. J. (2003). Effects of think aloud on translation speed, revision and segmentation. In Alves, F. (Eds), Triangulation Translation. Perspective in Process Orientated Research, (pp. 69-96). Amsterdam \& Philadelphia: John Benjamins.

Konings, M. J., Foulsham, T., Micklewright, D., \& Hettinga, F. J. (2019). Athlete-Opponent Interdependency Alters Pacing and Information-seeking Behavior. Medicine \& Science in Sports \& Exercise, Published Ahead of Print, doi: 10.1249/MSS.0000000000002101.

Kay, D., Marino, F.E., Cannon, J., St Clair Gibson, A., Lambert, M.I., \& Noakes, T.D. (2001). Evidence for neuromuscular fatigue during high-intensity cycling in warm, humid conditions. European Journal of Applied Physiology, 90, 411-419.

Konings, M. J., \& Hettinga, F. J. (2018). Pacing decision making in sport and the effects of interpersonal competition: A critical review. Sports Medicine, 48; 1829-1843. https://doi.org/10.1007/s40279-0180937-x.

Krings, H. P. (2001). Repairing texts. Empirical investigations of machine-translation post-editing processes. Kent, Ohio: Kent State University Press.

LaCaille, R. A., Masters, K. S., \& Heath, E. M. (2004). Effects of cognitive strategy and exercise setting on running performance, perceived exertion, affect, and satisfaction. Psychology of Sport and Exercise, 5(4), 461-476. https://doi.org/10.1016/S1469-0292(03)00039-6. 
Expertise differences in cognitions, gaze behaviour and cycling performance

928

929

930

931

932

933

934

935

936

937

938

939

940

941

942

943

944

945

946

947

948

949

Lima-Silva, A. E., Correia-Oliveira, C. R., Tenorio, L., Melo, A. A., Bertuzzi, R., \& Bishop, D. (2013). Prior exercise reduces fast-start duration and end-spurt magnitude during cycling time-trial. International Journal of Sports Medicine, 34(8), 736-741. doi:10.1055/s-0032-1331258.

MacPhail, C., Khoza, N., Abler, L., \& Ranganathan, M. (2016). Process guidelines for establishing Intercoder Reliability in qualitative studies. Qualitative Research, 16, 198-212. https://doi.org/10.1177/1468794115577012.

Mann, D. T., Williams, A. M., Ward, P., \& Janelle, C. M. (2007). Perceptual-cognitive expertise in sport: A meta-analysis. Journal of Sport and Exercise Psychology, 29(4), 457-478. https://doi.org/10.1123/jsep.29.4.457.

Marcora, S. M. (2008). Do we really need a central governor to explain brain regulation of exercise performance? European Journal of Applied Physiology, 104(5): 929-931. https://doi.org/10.1007/s00421-008-0818-3.

McCormick, A., Meijen, C., Marcora, S. M. (2015). Psychological Determinants of Whole-Body Endurance Performance. Sports Medicine, 45(7): 997-1015. https://doi.org/10.1007/s40279-0150319-6PMID:25771784.

McPherson, S. L., \& Kernodle, M. (2007). Mapping two new points on the tennis expertise continuum: Tactical skills of adult advanced beginners and entry-level professionals during competition. Journal of Sports Sciences, 25(8), 945-959. http://dx.doi.org/10. 1080/02640410600908035.

Mestre, D. R., Maïano, C., Dagonneau, V., \& Mercier, C. S. (2011). Does virtual reality enhance exercise performance, enjoyment, and dissociation? An exploratory study on a stationary bike apparatus. $\begin{array}{lllll}\text { Presence: } \quad \text { Teleoperators and } & \text { Virtual }\end{array}$ https://doi.org/10.1162/pres_a_00031. 
Expertise differences in cognitions, gaze behaviour and cycling performance

950

951

952

953

954

955

956

957

958

959

960

961

962

963

964

965

966

967

968

969

970

971

972

973

Micklewright, D., Papadopoulou, E., Swart, J., \& Noakes, T. (2010). Previous experience influences pacing during $20 \mathrm{~km}$ time trial cycling. British Journal of Sports Medicine, 44(13), 952-960. http://dx.doi.org/10.1136/bjsm.2009.057315.

Morgan, W. P., \& Pollock, M. L. (1977). Psychologic characterization of the elite distance runner. Annals of the New York Academy of Sciences, 301, 382-403. https://doi.org/10.1111/j.17496632.1977.tb38215.x.

Nicholls, A. R., \& Polman, R. C. J. (2008). Think aloud: acute stress and coping strategies during golf performances. Anxiety, Stress and Coping, 21, 283-294. https://doi.org/10.1080/10615800701609207.

Nietfeld, J. L. (2003). An examination of metacognitive strategy use and monitoring skills by competitive middle distance runners. Journal of Applied Sport Psychology, 15, 307-320. https://doi.org/10.1080/714044199.

Samson, A., Simpson, D., Kamphoff, C., \& Langlier, A. (2015). Think aloud: An examination of distance runners' thought processes. International Journal of Sport and Exercise Psychology, 15(2), 176-189. https://doi.org/10.1080/1612197X.2015.1069877.

Schmit, C., \& Brisswalter, J. (2018). Executive functioning during prolonged exercise: a fatigue-based neurocognitive perspective. International Review of Sport and Exercise Psychology, 1-19. https://doi.org/10.1080/1750984X.2018.1483527.

Schweizer, G., \& Furley, P. (2016). Reproducible research in sport and exercise psychology: The role of sample sizes. Psychology of Sport and Exercise, 23, 114-122. http://dx.doi.org/10.1016/j.psychsport.2015.11.005.

Simpson, D., Post, P. G., Young, G., \& Jensen, P. R. (2014). "It's not about taking the easy road": The experiences of ultramarathon runners. The Sport Psychologist, 28(2), 176-185. http://dx.doi.org/10.1123/tsp.2013-0064. 
Expertise differences in cognitions, gaze behaviour and cycling performance

974

975

976

977

978

979

980

981

982

983

984

985

986

987

988

989

990

991

992

993

994

995

996

SMI (SensoMotoric Instruments) (2012). BeGazeTM Manual Version 3.1.

Smits, B. L. M., Pepping, G. J., \& Hettinga, F. J. (2014). Pacing and decision making in sport and exercise: the roles of perception and action in the regulation of exercise intensity. Sports Medicine, 44(6), 763775. https://doi.org/10.1007/s40279-014-0163-0.

Stock, J. \& Cervone, D. (1990). Proximal goal-setting and self-regulatory processes. Cognitive Therapy and Research, 14(5), 483-498. https://doi.org/10.1007/BF01172969.

Stone, M. R., Thomas, K., Wilkinson, M., Jones, A. M., St Clair Gibson, A. \& Thompson, K. G. (2012). Effects of deception on exercise performance: Implications for determinants of fatigue in humans. Medicine and Science in Sports and Exercise, 44(3), 534-541. doi: 10.1249/MSS.0b013e318232cf77.

St Clair Gibson, A., Lambert, E. V., Rauch, L. H. G., Tucker, R., Baden, D. A., Foster, C., \& Noakes, T. D. (2006). The role of information processing between the brain and peripheral physiological systems in pacing and perception of effort. Sports Medicine, 36(8), 705-722. https://doi.org/10.2165/00007256200636080-00006.

Taylor, D., \& Smith, M. F. (2013). Scalar-linear increases in perceived exertion are dissociated from residual physiological responses during sprint-distance triathlon. Physiology and Behavior, 118, 178184. http://dx.doi.org/10.1016/j.physbeh.2013.05.031.

Triplett, N. (1898). The Dynamogenic Factors in Pacemaking and Competition. The American Journal of Psychology, 9(4), 507-533. doi: 10.2307/1412188.

Ulmer, H. V. (1996) Concept of an extracellular regulation of muscular metabolic rate during heavy exercise in humans by psychophysiological feedback, Experientia, 52, 416-420. https://doi.org/10.1007/BF01919309.

Vansteenkiste, M., Lens, W., Elliot, A., Soenens, B., \& Mouratidis, A. (2014). Moving the achievement goal approach one step forward: Toward a systematic examination of the autonomous and controlled 
Expertise differences in cognitions, gaze behaviour and cycling performance

997 reasons underlying achievement goals. Educational Psychologist, 49, 153-174. $998 \quad$ http://dx.doi.org/10.1080/00461520.2014.928598.

999 Van der Kamp, J., Rivas, F., Van Doorn, H., \& Savelsbergh, G. (2008). Ventral and dorsal system 1000 contributions to visual anticipation in fast ball sports. International Journal of Sport Psychology, 39(2),

1001 100.

1002

Van Gog, T., Paas, F., van Merriënboer, J. J., \& Witte, P. (2005). Uncovering the problem-solving 1003 process: Cued retrospective reporting versus concurrent and retrospective reporting. Journal of 1004 Experimental Psychology: Applied, 11(4), 237. http://dx.doi.org/10.1037/1076-898X.11.4.237.

1005

Vater, C., Williams, A. M., \& Hossner, E. J. (2019). What do we see out of the corner of our eye? The 1006 role of visual pivots and gaze anchors in sport. International Review of Sport and Exercise Psychology,

1007 1-23. https://doi.org/10.1080/1750984X.2019.1582082.

Vickers, J. N. (2007). Perception, Cognition and Decision Training: The Quiet Eye in Action. Champaign, 1009 Illinois: Human Kinetics.

1010 Voss, M. W., Kramer, A. F., Basak, C., Parkash, R. S., \& Roberts, B. (2010). Are expert athletes 'expert' 1011 in the cognitive laboratory? A meta-analytic review of cognition and sport expertise. Applied Cognitive 1012 Psychology, 24, 812-826. https://doi.org/10.1002/acp.1588.

1013

Weinberg, R., Miller, A., \& Horn, T. (2012). The influence of a self-talk intervention on collegiate cross1014 country runners. International Journal of Sport and Exercise Psychology, 10(2), 123-134. 1015 https://doi.org/10.1080/1612197X.2012.645135. (2017). Changes in cognition over a $16.1 \mathrm{~km}$ cycling time trial using a think aloud protocol: Preliminary evidence. International Journal of Sport and Exercise Psychology, 173(3), 266-274. https://doi.org/10.1080/1612197X.2017.1292302. 
Expertise differences in cognitions, gaze behaviour and cycling performance

1020

1021

1022

1023

1024

1025

1026

1027

1028

1029

1030

1031

1032

1033

1034

1035

1036

1037

1038

1039

1040

Whitehead, A. E., Jones, H. S., Williams, E. L., Rowley, C., Quayle, L., Marchant, D. C., \& Polman, R. C. J. (2018). Investigating the relationship between cognitions, pacing strategies and performance in 16.1 km cycling time trials using a think aloud protocol. Psychology of Sport \& Exercise, 34, 95 - 109. https://doi.org/10.1016/j.psychsport.2017.10.001.

Whitehead, A. E., Taylor, J. A., \& Polman, R. C. J. (2015). Examination of the suitability of collecting in event cognitive processes using Think Aloud protocol in golf. Frontiers in Psychology, 6, 1083. https://doi.org/10.3389/fpsyg.2015.01083.

Williams, D. M. (2008). Exercise, affect, and adherence: an integrated model and a case for self-paced

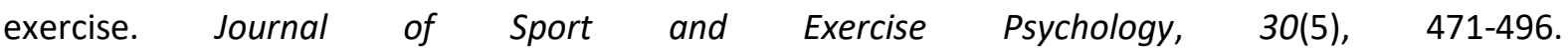
https://doi.org/10.1123/jsep.30.5.471.

Williams, A. M., Davids, K., \& Williams, J. G. (1999). Visual perception and action in sport. London: E. \& F.N. Spon.

Williams, A. M., \& Ford, P. R. (2008). Expertise and expert performance in sport. International Review of Sport and Exercise Psychology, 1, 4-18. https://doi.org/10.1080/17509840701836867.

Williams, E. L., Jones, H. S., S. Sparks, A., Marchant, D. C., Micklewright, D., \& McNaughton, L. R. (2014). Deception studies manipulating centrally acting performance modifiers: A review. Medicine and Science in Sports and Exercise, 46(7), 1441-1451. http://dx.doi. org/10.1249/MSS.0000000000000235.

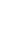


Table 1: Description of primary and secondary themes from the Think Aloud verbalisations.

\begin{tabular}{|c|c|c|c|}
\hline $\begin{array}{l}\text { Secondary } \\
\text { Themes }\end{array}$ & $\begin{array}{l}\text { Primary } \\
\text { Themes }\end{array}$ & Description & Example of raw data quotes \\
\hline \multirow[t]{4}{*}{$\begin{array}{l}\text { Internal Sensory } \\
\text { Monitoring }\end{array}$} & Breathing & $\begin{array}{l}\text { Reference to breathing or respiratory } \\
\text { regulation }\end{array}$ & $\begin{array}{l}\text { "Control my breathing." (Trained P3) } \\
\text { "Just keep breathing" (Untrained P11) }\end{array}$ \\
\hline & $\begin{array}{l}\text { Pain and } \\
\text { Discomfort }\end{array}$ & $\begin{array}{l}\text { Reference to physical or mental pain and } \\
\text { fatigue and general discomfort during the task }\end{array}$ & $\begin{array}{l}\text { "It's starting to hurt a bit now." (Trained P4) } \\
\text { "I can feel the burn on that straight away." (Untrained P12) }\end{array}$ \\
\hline & Hydration & Reference to taking or needing a drink & $\begin{array}{l}\text { "Bit of water. Bit of dry mouth". (Trained P7) } \\
\text { "Water would be good, water would be good now." (Untrained P14) }\end{array}$ \\
\hline & Heart Rate & $\begin{array}{l}\text { Increasing or decreasing of heart rate, or } \\
\text { statement of heart rate value }\end{array}$ & $\begin{array}{l}\text { "Heart rate's fairly consistent" (Trained P1) } \\
\text { "That's sending my pulse rate up higher" (Untrained P15) }\end{array}$ \\
\hline \multirow[t]{5}{*}{$\begin{array}{l}\text { Active Self- } \\
\text { Regulation }\end{array}$} & Cadence & Verbalisations relating to pedal stroke & $\begin{array}{l}\text { "Cadence is steady" (Trained P9) } \\
\text { "Just try and lower the RPM a little bit" (Untrained P13) }\end{array}$ \\
\hline & Speed & Reference relating specifically to speed & $\begin{array}{l}\text { "Speed has dropped" (Trained P1) } \\
\text { "I'll try and keep KPH more consistent, but it seems to fluctuate between } \\
\text { sort of } 31 \text { and } 33 \text { " (Untrained P18) }\end{array}$ \\
\hline & Power & Reference relating to power output or watts & $\begin{array}{l}\text { "That's it Just need to get to the top of that hill. Keep your wattage up" } \\
\text { (Trained P3) } \\
\text { "I'm trying to keep these watts, like, consistent. It's very difficult" } \\
\text { (Untrained P10) }\end{array}$ \\
\hline & Pace & $\begin{array}{l}\text { Reference to purposeful strategy or action- } \\
\text { based changes to pace }\end{array}$ & $\begin{array}{l}\text { "Not too hard. Get the pace right. Keep it steady. That's it" (Trained P8) } \\
\text { "yeah, that's going to get me there, just around } 32 \text { minutes. So this is } \\
\text { about the pace I want" (Untrained P17) }\end{array}$ \\
\hline & Gear use & Reference to gear change or gear selection & $\begin{array}{l}\text { "Just knock the gear back" (Trained P6) } \\
\text { "Er, I think I was at quite a high gear last time so I'm just trying to take it a } \\
\text { bit slower and then hopefully have a bit more as it goes on" (Untrained } \\
\text { P12) }\end{array}$ \\
\hline
\end{tabular}


Motivation Verbalisations relating to self-motivation or
positive encouragement

Technique Reference to technique including body position and coaching points
"Keep it going, come on" (Trained P9)

"Come on, go on lad" (Untrained P15)

"Slightly forward on the saddle" (Trained P5)

"Just try and think about how I pedal as well and if I can be more economical or efficient" (Untrained, P16)

\begin{tabular}{|c|c|c|c|}
\hline \multirow[t]{3}{*}{$\begin{array}{l}\text { Outward } \\
\text { Monitoring }\end{array}$} & Time & $\begin{array}{l}\text { Reference to time, time elapsed or expected } \\
\text { finish time }\end{array}$ & $\begin{array}{l}\text { "4 minutes gone" (Trained P2) } \\
\text { "So I know I've got } 10 \text { minutes to do 6K now" (Untrained P17) }\end{array}$ \\
\hline & Distance & $\begin{array}{l}\text { Any reference to distance covered or distance } \\
\text { remaining }\end{array}$ & $\begin{array}{l}\text { "Nearly a third of the way there" (Trained P7) } \\
\text { "So, I have } 14 \mathrm{~km} \text { left" (Untrained P10) }\end{array}$ \\
\hline & Competitor* & Reference to the virtual avatar & $\begin{array}{l}\text { "The avatar's still a bit ahead of me" (Trained P1) } \\
\text { "So just by focussing on him that's made me speed up trying to catch him" } \\
\text { (Untrained P14) }\end{array}$ \\
\hline \multirow[t]{2}{*}{ Distraction } & $\begin{array}{l}\text { Irrelevant } \\
\text { Information }\end{array}$ & Verbalisations not relevant to the given task & $\begin{array}{l}\text { "Had a bit of a cough this morning in the pool, I hope I'm not getting a } \\
\text { cold. Stupid English weather" (Trained P2) } \\
\text { "just got the foo fighters in my head, it was the last song I heard on the } \\
\text { radio before finishing work. Just got that going on in my head right now" } \\
\text { (Untrained P11) }\end{array}$ \\
\hline & $\begin{array}{l}\text { Course } \\
\text { Scenery }\end{array}$ & $\begin{array}{l}\text { Reference to the visual display of the simulated } \\
\text { course, avatar or scenery }\end{array}$ & $\begin{array}{l}\text { "They keep sending these same trees back at me, time and time again. } \\
\text { They look very familiar" (Trained P5) } \\
\text { "The scenery is a bit distracting, not going past as quickly as I feel I'm } \\
\text { going, that's a bit odd" (Untrained P16) }\end{array}$ \\
\hline
\end{tabular}

* relevant to Study 2 only 
Table 2: Mean (SD) whole-trial performance data for trained and untrained groups during a $16.1 \mathrm{~km}$ time trial

1049

1050

\begin{tabular}{lll}
\hline & Trained & Untrained \\
\hline Time (mins) & $25.96 \pm 1.33^{*}$ & $30.75 \pm 2.44$ \\
Speed (km.hr-1) & $37.8 \pm 1.95 *$ & $31.68 \pm 2.60$ \\
Power Output (W) & $272 \pm 38^{*}$ & $180 \pm 38$ \\
Peak Power Output (W) & $285 \pm 42$ & $191 \pm 35$ \\
Cadence (rpm) & $95 \pm 9 *$ & $85 \pm 5$ \\
Heart Rate (beats.min $\left.{ }^{-1}\right)$ & $159 \pm 13$ & $142 \pm 25$
\end{tabular}

1051

* denotes significantly faster/greater values than the untrained group

1052

1053

1054

1055

1056

1057

1058

1059

1060

1061

1062

1063

1064

1065

1066

1067

1068

1069

1070

1071

1072

1073

1074

1075

1076

1077

1078

1079

1080

1081

1082

1083 
1087

1088

Table 3: Mean (SD) percentage of verbalisations for secondary themes for trained and untrained groups during a cycling time trial.

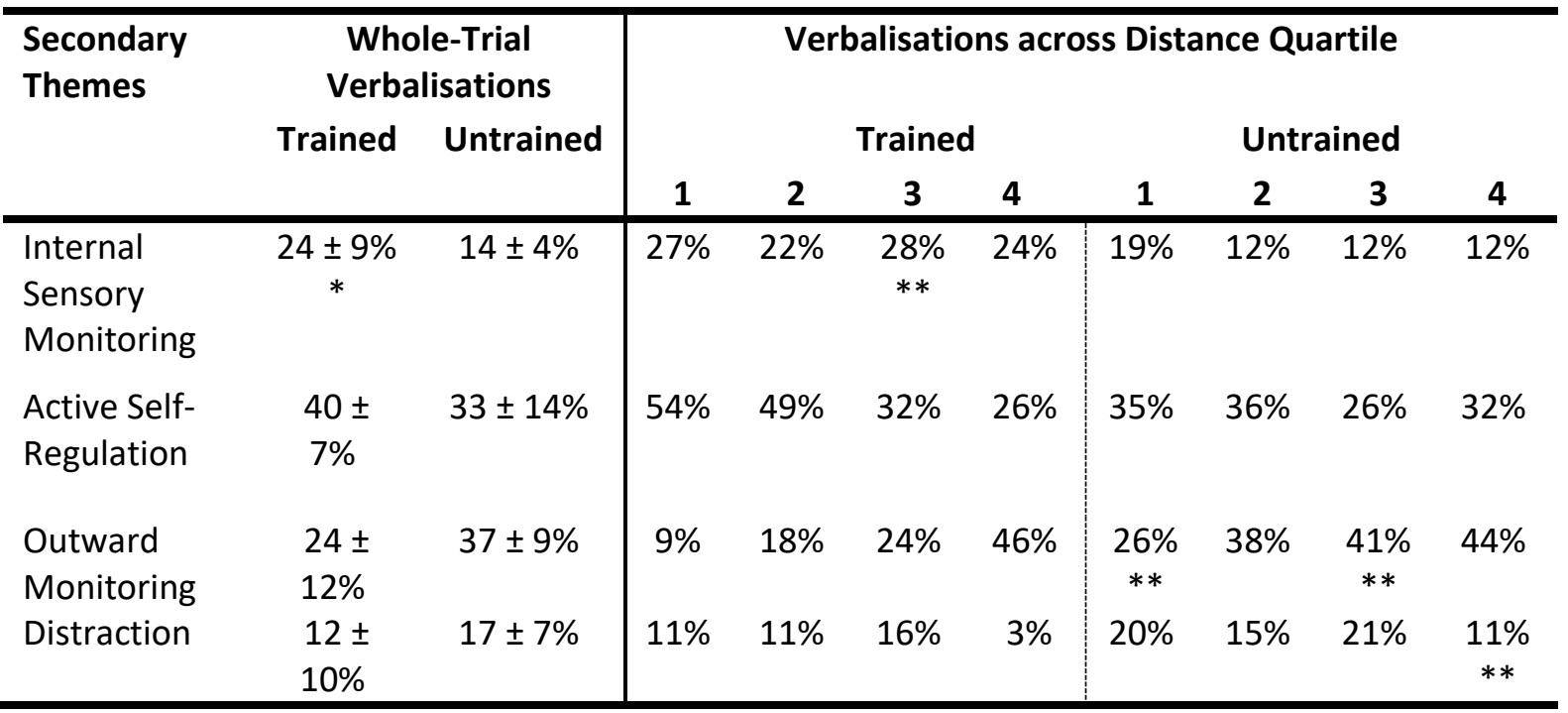

* denotes significantly more verbalisations than the other group as a whole trial

** denotes significantly more verbalisations than the other group at the distance quartile

Table 4: Between-group comparisons of primary themes verbalised across TT distance quartile.

\begin{tabular}{llcccccc}
\hline $\begin{array}{l}\text { Secondary } \\
\text { Theme }\end{array}$ & $\begin{array}{l}\text { Primary } \\
\text { Theme }\end{array}$ & $\begin{array}{c}\text { Distance } \\
\text { Quartile }\end{array}$ & $\begin{array}{c}\text { Mann- } \\
\text { Whitney } \\
(\mathbf{U})\end{array}$ & $\begin{array}{c}\text { Effect } \\
\text { Size } \\
(\mathbf{r})\end{array}$ & $\begin{array}{c}\text { Signf. } \\
(\boldsymbol{p})\end{array}$ & $\begin{array}{c}\text { Mean Ranks } \\
\text { Trained }\end{array}$ & Untrained \\
\hline $\begin{array}{l}\text { Internal } \\
\text { Sensory }\end{array}$ & Heart Rate & 3 & 7.00 & 0.56 & .044 & 9.33 & 5.00 \\
Monitoring & Hydration & 3 & 9.00 & 0.58 & .036 & 5.00 & 8.71 \\
& $\begin{array}{l}\text { Pain and } \\
\text { Active Self- }\end{array}$ & 3 & 7.00 & 0.57 & .039 & 9.33 & 5.00 \\
Regulation & Power & 2 & 6.00 & 0.63 & .24 & 9.50 & 4.86 \\
Outward & Pace & 2 & 5.00 & 0.64 & .020 & 4.33 & 9.29 \\
Monitoring & Time & 3 & 7.00 & 0.56 & .042 & 4.67 & 9.00 \\
Distraction & Distance & 1 & 4.00 & 0.68 & .015 & 4.17 & 9.43 \\
& Course & 1 & 8.00 & 0.56 & .043 & 4.83 & 8.86 \\
& Scenery & 2 & 9.00 & 0.58 & .036 & 5.00 & 8.71 \\
& & 4 & 8.00 & 0.56 & .043 & 4.83 & 8.86 \\
\hline
\end{tabular}


Expertise differences in cognitions, gaze behaviour and cycling performance

1095

Table 5: Within-group comparisons of primary themes verbalised across TT distance quartiles.

1098

\begin{tabular}{|c|c|c|c|c|c|c|}
\hline \multirow{2}{*}{$\begin{array}{l}\text { Secondary } \\
\text { Theme }\end{array}$} & \multirow{2}{*}{$\begin{array}{l}\text { Primary } \\
\text { Theme }\end{array}$} & \multirow[t]{2}{*}{ Group } & \multicolumn{2}{|c|}{ Quartile Difference } & \multicolumn{2}{|c|}{ Post-Hoc Analysis } \\
\hline & & & & $\begin{array}{l}\text { Wilcoxon } \\
\text { Rank (Z) }\end{array}$ & $\begin{array}{l}\text { Effect } \\
\text { Size }(r)\end{array}$ & $\begin{array}{l}\text { Signf. } \\
\text { (p) }\end{array}$ \\
\hline \multirow[t]{7}{*}{$\begin{array}{l}\text { Active Self- } \\
\text { Regulation }\end{array}$} & Motivation & Untrained & $\begin{array}{l}\text { Quartile } 1 \text { - } \\
\text { Quartile } 4 \text { * }\end{array}$ & -2.02 & 0.58 & .043 \\
\hline & & & $\begin{array}{l}\text { Quartile 2- } \\
\text { Quartile 4* }\end{array}$ & -2.02 & 0.58 & .043 \\
\hline & & & $\begin{array}{l}\text { Quartile } 3 \text { - } \\
\text { Quartile } 4 \text { * }\end{array}$ & -2.02 & 0.58 & .043 \\
\hline & Power & Trained & $\begin{array}{l}\text { Quartile } 2 * \text { - } \\
\text { Quartile } 3\end{array}$ & -2.02 & 0.58 & .043 \\
\hline & & & $\begin{array}{l}\text { Quartile 2*_ } \\
\text { Quartile } 4\end{array}$ & -1.99 & 0.57 & .046 \\
\hline & Pace & Untrained & $\begin{array}{l}\text { Quartile } 1^{*}- \\
\text { Quartile } 3\end{array}$ & -2.02 & 0.58 & .043 \\
\hline & & & $\begin{array}{l}\text { Quartile } 1{ }^{*} \text { - } \\
\text { Quartile } 4\end{array}$ & -2.02 & 0.58 & .043 \\
\hline \multirow[t]{6}{*}{$\begin{array}{l}\text { Outward } \\
\text { Monitoring }\end{array}$} & Distance & Trained & $\begin{array}{l}\text { Quartile 1- } \\
\text { Quartile 3* }\end{array}$ & -2.20 & 0.63 & .028 \\
\hline & & & $\begin{array}{l}\text { Quartile } 1 \text { - } \\
\text { Quartile } 4 \text { * }\end{array}$ & -2.20 & 0.63 & .028 \\
\hline & & & $\begin{array}{l}\text { Quartile } 2 \text { - } \\
\text { Quartile } 4 \text { * }\end{array}$ & -2.20 & 0.63 & .028 \\
\hline & & & $\begin{array}{l}\text { Quartile } 3 \text { - } \\
\text { Quartile } 4 \text { * }\end{array}$ & -2.02 & 0.58 & .043 \\
\hline & & Untrained & $\begin{array}{l}\text { Quartile } 1 \text { - } \\
\text { Quartile } 3 \text { * }\end{array}$ & -2.20 & 0.63 & .028 \\
\hline & & & $\begin{array}{l}\text { Quartile } 1 \text { - } \\
\text { Quartile 4* }\end{array}$ & -2.37 & 0.69 & .018 \\
\hline
\end{tabular}

* denotes the significantly higher distance quartile

1101 
Expertise differences in cognitions, gaze behaviour and cycling performance

Table 6: Mean (SD) percentage dwell time for each area of interest across TT distance quartiles.

\begin{tabular}{|c|c|c|c|c|c|c|c|c|c|}
\hline \multirow[t]{3}{*}{ Area of Interest } & \multirow[t]{3}{*}{ Group } & \multicolumn{8}{|c|}{ Distance Quartile } \\
\hline & & \multicolumn{2}{|l|}{1} & \multicolumn{2}{|c|}{2} & \multicolumn{2}{|l|}{3} & \multicolumn{2}{|l|}{4} \\
\hline & & Mean & SD & Mean & SD & Mean & SD & Mean & SD \\
\hline \multirow[t]{3}{*}{ Screen } & Untrained & 27.99 & 13.32 & 23.24 & 16.56 & 19.72 & 13.50 & 13.56 & 11.01 \\
\hline & Trained & 23.85 & 21.25 & 33.43 & 25.34 & 27.69 & 24.03 & 15.40 & 14.01 \\
\hline & Total & 26.08 & 16.78 & 27.94 & 20.80 & 23.40 & 18.67 & 14.41 & 11.97 \\
\hline \multirow[t]{3}{*}{ Power } & Untrained & 3.34 & 2.33 & 4.76 & 5.48 & 4.01 & 3.36 & 2.86 & 3.80 \\
\hline & Trained & 22.48 & 21.72 & 14.42 & 14.97 & 11.27 & 9.33 & 5.01 & 5.09 \\
\hline & Total & 12.18 & 17.26 & 9.22 & 11.55 & 7.36 & 7.49 & 3.85 & 4.39 \\
\hline \multirow[t]{3}{*}{ Cadence } & Untrained & 3.14 & 3.08 & 2.30 & 2.60 & 2.31 & 3.44 & 0.95 & 1.13 \\
\hline & Trained & 3.07 & 2.35 & 1.45 & 0.82 & 1.32 & 1.13 & 0.94 & 0.95 \\
\hline & Total & 3.10 & 2.65 & 1.90 & 1.96 & 1.86 & 2.59 & 0.94 & 1.00 \\
\hline \multirow[t]{3}{*}{ Heart Rate } & Untrained & 4.06 & 3.34 & 3.64 & 2.88 & 3.63 & 3.26 & 2.73 & 2.74 \\
\hline & Trained & 2.91 & 1.94 & 1.50 & 1.60 & 2.20 & 2.28 & 1.77 & 1.93 \\
\hline & Total & 3.53 & 2.74 & 2.66 & 2.54 & 2.97 & 2.83 & 2.29 & 2.36 \\
\hline \multirow[t]{3}{*}{ Distance } & Untrained & 5.37 & 4.21 & 5.59 & 3.89 & 7.84 & 5.98 & 7.03 & 5.80 \\
\hline & Trained & 1.70 & 0.97 & 2.02 & 1.08 & 2.93 & 1.57 & 2.66 & 1.66 \\
\hline & Total & 3.67 & 3.59 & 3.95 & 3.39 & 5.58 & 5.04 & 5.01 & 4.81 \\
\hline \multirow[t]{3}{*}{ Speed } & Untrained & 2.97 & 4.12 & 3.17 & 4.37 & 2.57 & 2.34 & 1.74 & 1.89 \\
\hline & Trained & 0.42 & 0.50 & 0.23 & 0.26 & 0.14 & 0.17 & 0.51 & 0.46 \\
\hline & Total & 1.79 & 3.22 & 1.81 & 3.45 & 1.45 & 2.09 & 1.17 & 1.51 \\
\hline \multirow[t]{3}{*}{ Time } & Untrained & 1.10 & 1.15 & 1.06 & 1.42 & 1.02 & 1.34 & 1.04 & 1.33 \\
\hline & Trained & 0.29 & 0.30 & 0.53 & 0.52 & 0.54 & 0.77 & 0.38 & 0.30 \\
\hline & Total & 0.73 & 0.94 & 0.82 & 1.09 & 0.80 & 1.10 & 0.73 & 1.02 \\
\hline
\end{tabular}


Table 7: Mean (SD) gaze frequency for each area of interest across TT distance quartiles.

\begin{tabular}{|c|c|c|c|c|c|c|c|c|c|}
\hline \multirow[t]{3}{*}{ Area of Interest } & \multirow[t]{3}{*}{ Group } & \multicolumn{8}{|c|}{ Distance Quartile } \\
\hline & & \multicolumn{2}{|l|}{1} & \multicolumn{2}{|l|}{2} & \multicolumn{2}{|l|}{3} & \multicolumn{2}{|l|}{4} \\
\hline & & Mean & SD & Mean & SD & Mean & SD & Mean & SD \\
\hline \multirow[t]{3}{*}{ Screen } & Untrained & 540.71 & 225.62 & 408.00 & 223.23 & 397.00 & 257.83 & 275.00 & 245.94 \\
\hline & Trained & 336.83 & 316.17 & 491.50 & 384.87 & 449.33 & 393.13 & 287.00 & 258.45 \\
\hline & Total & 446.62 & 279.81 & 446.54 & 297.51 & 421.15 & 313.64 & 280.54 & 241.07 \\
\hline \multirow[t]{3}{*}{ Power } & Untrained & 58.00 & 36.96 & 68.86 & 47.93 & 72.43 & 54.70 & 50.86 & 40.01 \\
\hline & Trained & 324.00 & 327.00 & 215.33 & 285.51 & 171.17 & 191.12 & 71.33 & 76.83 \\
\hline & Total & 180.77 & 253.55 & 136.46 & 202.21 & 118.00 & 139.07 & 60.31 & 58.07 \\
\hline \multirow[t]{3}{*}{ Cadence } & Untrained & 61.57 & 55.35 & 43.43 & 44.96 & 46.57 & 55.62 & 13.00 & 7.57 \\
\hline & Trained & 53.50 & 46.62 & 22.17 & 10.03 & 21.83 & 15.89 & 18.67 & 19.00 \\
\hline & Total & 57.85 & 49.55 & 33.62 & 34.27 & 35.15 & 42.62 & 15.62 & 13.70 \\
\hline \multirow[t]{3}{*}{ Heart Rate } & Untrained & 81.00 & 50.52 & 73.43 & 55.64 & 75.57 & 54.01 & 56.57 & 53.83 \\
\hline & Trained & 52.17 & 33.53 & 22.33 & 22.42 & 39.00 & 43.25 & 30.33 & 32.67 \\
\hline & Total & 67.69 & 44.37 & 49.85 & 49.60 & 58.69 & 50.97 & 44.46 & 45.60 \\
\hline \multirow[t]{3}{*}{ Distance } & Untrained & 88.86 & 63.91 & 110.71 & 70.32 & 155.29 & 116.53 & 136.57 & 120.77 \\
\hline & Trained & 27.83 & 15.66 & 29.67 & 19.27 & 50.00 & 29.31 & 44.50 & 24.51 \\
\hline & Total & 60.69 & 56.10 & 73.31 & 66.30 & 106.69 & 100.66 & 94.08 & 99.12 \\
\hline
\end{tabular}


Expertise differences in cognitions, gaze behaviour and cycling performance

$\begin{array}{llllllllll}\text { Speed } & \text { Untrained } & 61.57 & 85.23 & 69.57 & 114.09 & 56.29 & 63.49 & 36.57 & 44.17 \\ & \text { Trained } & 6.67 & 5.75 & 3.50 & 4.18 & 3.00 & 3.16 & 8.00 & 7.43 \\ & \text { Total } & 36.23 & 66.76 & 39.08 & 87.70 & 31.69 & 52.76 & 23.38 & 34.91 \\ \text { Time } & \text { Untrained } & 24.57 & 24.28 & 24.43 & 31.09 & 20.86 & 23.00 & 24.29 & 33.05 \\ & \text { Trained } & 4.17 & 3.31 & 9.17 & 7.68 & 8.17 & 7.96 & 7.17 & 5.27 \\ & \text { Total } & 15.15 & 20.28 & 17.38 & 23.89 & 15.00 & 18.28 & 16.38 & 25.23\end{array}$

1116

1117

1118

1119

1120

1121

1122

1123

1124
1125

1126

1127

\begin{tabular}{lcccc}
\hline & \multicolumn{2}{c}{ Trained } & \multicolumn{2}{c}{ Untrained } \\
& ALONE & COMP & ALONE & COMP \\
\hline Time (mins) & $25.94 \pm 1.21^{*}$ & $25.68 \pm 1.03^{*}$ & $31.53 \pm 2.73$ & $31.75 \pm 3.00$ \\
Speed (km.hr ${ }^{-1}$ ) & $37.4 \pm 1.7$ & $37.6 \pm 1.4$ & $31.4 \pm 2.2$ & $31.2 \pm 2.6$ \\
Power Output (W) & $273 \pm 30^{*}$ & $277 \pm 26^{*}$ & $177 \pm 37$ & $177 \pm 40$ \\
Peak Power Output (W) & $286 \pm 29$ & $283 \pm 29$ & $191 \pm 58$ & $191 \pm 50$ \\
Cadence (rpm) & $90 \pm 7^{*}$ & $92 \pm 8 *$ & $80 \pm 6$ & $79 \pm 8$ \\
Heart Rate (beats.min $\left.{ }^{-1}\right)$ & $146 \pm 22$ & $163 \pm 14$ & $151 \pm 15$ & $147 \pm 25$ \\
\hline
\end{tabular}

1128

* denotes significantly faster/greater values than the untrained group

Table 8: Mean (SD) whole-trial performance data for trained and untrained groups during alone and

competitor trials.

1129

1130 
1131

1132

1133

1134

1135

1136

1137

1138

1139

1140

1141

1142

1143

1144

1145

1146

1147

1148

1149

1150

1151

1152

1153

1154

1155

\begin{tabular}{lcccc}
\hline Secondary Themes & \multicolumn{2}{c}{ Trained } & \multicolumn{2}{c}{ Untrained } \\
& ALONE & COMP & ALONE & COMP \\
\hline Internal Sensory Monitoring & $19 \pm 19 \%$ & $12 \pm 11 \%$ & $19 \pm 12 \%$ & $14 \pm 11 \%$ \\
Active Self-Regulation & $42 \pm 14 \%$ & $53 \pm 17 \% *$ & $41 \pm 10 \%$ & $38 \pm 9 \%$ \\
Outward Monitoring & $24 \pm 13 \%$ & $28 \pm 14 \%$ & $30 \pm 10 \%$ & $41 \pm 13 \%$ \\
Distraction & $15 \pm 13 \%$ & $7 \pm 9 \%$ & $10 \pm 10 \%$ & $7 \pm 8 \%$ \\
\hline
\end{tabular}

Table 9: Mean (SD) percentage of whole-trial verbalisations of secondary themes for trained and untrained groups during alone and competitor trials.

* denotes significantly higher percentage than the untrained group for the COMP trial 
Table 10: Between-group comparisons of primary themes verbalised during alone and competitor trials.

\begin{tabular}{lllccccc}
\hline $\begin{array}{l}\text { Secondary } \\
\text { theme }\end{array}$ & $\begin{array}{l}\text { Primary } \\
\text { theme }\end{array}$ & Trial & $\begin{array}{c}\text { Mann- } \\
\text { Whitney } \\
(\mathbf{U})\end{array}$ & $\begin{array}{c}\text { Effect } \\
\text { size } \\
(\mathbf{r})\end{array}$ & $\begin{array}{c}\text { Signf. } \\
(\boldsymbol{p})\end{array}$ & $\begin{array}{l}\text { Mean Rank data } \\
\text { Trained }\end{array}$ & Untrained \\
\hline $\begin{array}{l}\text { Active Self- } \\
\text { Regulation }\end{array}$ & Power & COMP & 7.00 & 0.63 & .015 & 10.63 & 5.00 \\
& Pace & COMP & 6.00 & 0.66 & .011 & 5.25 & 11.14 \\
& & ALONE & 6.00 & 0.64 & .013 & 4.50 & 10.33 \\
& Technique & ALONE & 9.00 & 0.55 & .033 & 5.00 & 10.00 \\
$\begin{array}{l}\text { Outward } \\
\text { Monitoring }\end{array}$ & Time & ALONE & 9.00 & 0.55 & .033 & 5.00 & 10.00 \\
\hline
\end{tabular}

1156

1157

1158

1159

1160

Table 11: Within-group comparisons of primary themes verbalised across TT distance quartiles during alone and competitor trials.

\begin{tabular}{|c|c|c|c|c|c|c|c|}
\hline \multirow{2}{*}{$\begin{array}{l}\text { Secondary } \\
\text { theme }\end{array}$} & \multirow{2}{*}{$\begin{array}{l}\text { Primary } \\
\text { theme }\end{array}$} & \multirow[t]{2}{*}{ Trial } & \multirow[t]{2}{*}{ Group } & \multirow[t]{2}{*}{ Quartile differer } & \multicolumn{3}{|c|}{ Post-hoc analysis } \\
\hline & & & & & $\begin{array}{l}\text { Wilcoxon } \\
\text { Rank (Z) }\end{array}$ & $\begin{array}{l}\text { Effect } \\
\text { Size (r) }\end{array}$ & $\begin{array}{l}\text { Signf. } \\
\text { (p) }\end{array}$ \\
\hline \multirow[t]{8}{*}{$\begin{array}{l}\text { Active Self- } \\
\text { Regulation }\end{array}$} & Motivation & ALONE & Untrained & $\begin{array}{l}\text { Quartile 1- } \\
\text { Quartile 3* }\end{array}$ & -1.99 & 0.63 & .046 \\
\hline & & & & $\begin{array}{l}\text { Quartile 1- } \\
\text { Quartile 4* }\end{array}$ & -2.37 & 0.75 & .018 \\
\hline & & & & $\begin{array}{l}\text { Quartile 2- } \\
\text { Quartile 4* }\end{array}$ & -2.02 & 0.64 & .028 \\
\hline & & ALONE & Trained & $\begin{array}{l}\text { Quartile 1- } \\
\text { Quartile 4* }\end{array}$ & -2.02 & 0.71 & .043 \\
\hline & & COMP & Trained & $\begin{array}{l}\text { Quartile 1- } \\
\text { Quartile 4* }\end{array}$ & -2.37 & 0.84 & .018 \\
\hline & & & & $\begin{array}{l}\text { Quartile 3- } \\
\text { Quartile 4* }\end{array}$ & -2.20 & 0.78 & .028 \\
\hline & Pace & COMP & Untrained & $\begin{array}{l}\text { Quartile 2* - } \\
\text { Quartile } 3\end{array}$ & -2.02 & 0.64 & .043 \\
\hline & & & & $\begin{array}{l}\text { Quartile 2*_- } \\
\text { Quartile } 4\end{array}$ & -2.02 & 0.64 & .028 \\
\hline \multirow[t]{3}{*}{$\begin{array}{l}\text { Outward } \\
\text { Monitoring }\end{array}$} & Distance & ALONE & Trained & $\begin{array}{l}\text { Quartile 1- } \\
\text { Quartile 3* }\end{array}$ & -2.20 & 0.78 & .028 \\
\hline & & & & $\begin{array}{l}\text { Quartile 1- } \\
\text { Quartile 4* }\end{array}$ & -2.20 & 0.78 & .043 \\
\hline & & & & $\begin{array}{l}\text { Quartile 2- } \\
\text { Quartile 3* }\end{array}$ & -2.20 & 0.78 & .028 \\
\hline
\end{tabular}


1163

1164

1165

1166

1167

1168

1169

1170

1171

1172

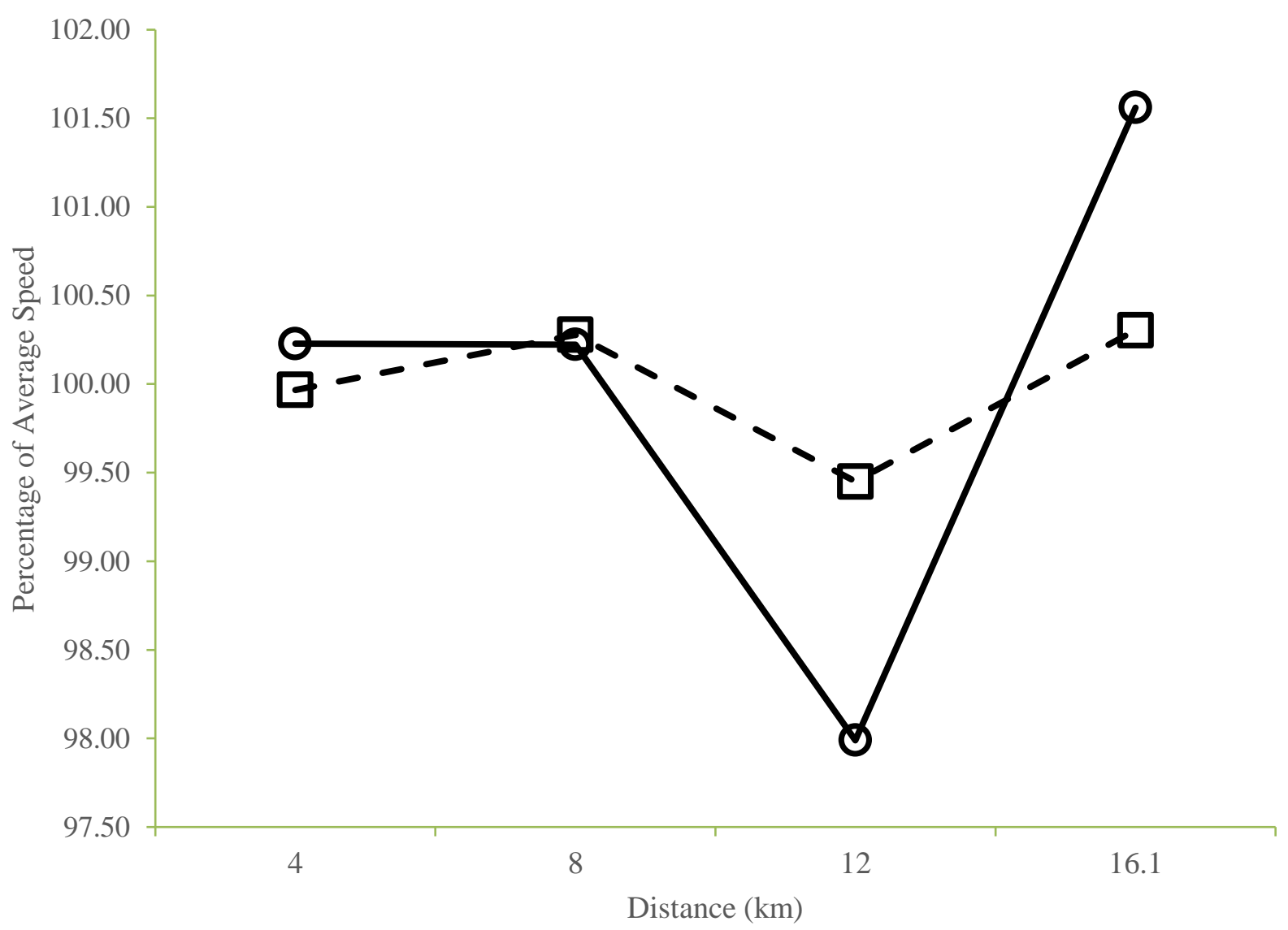

1173

Figure 1: Mean percentage of average speed for trained and untrained groups across the trial. 


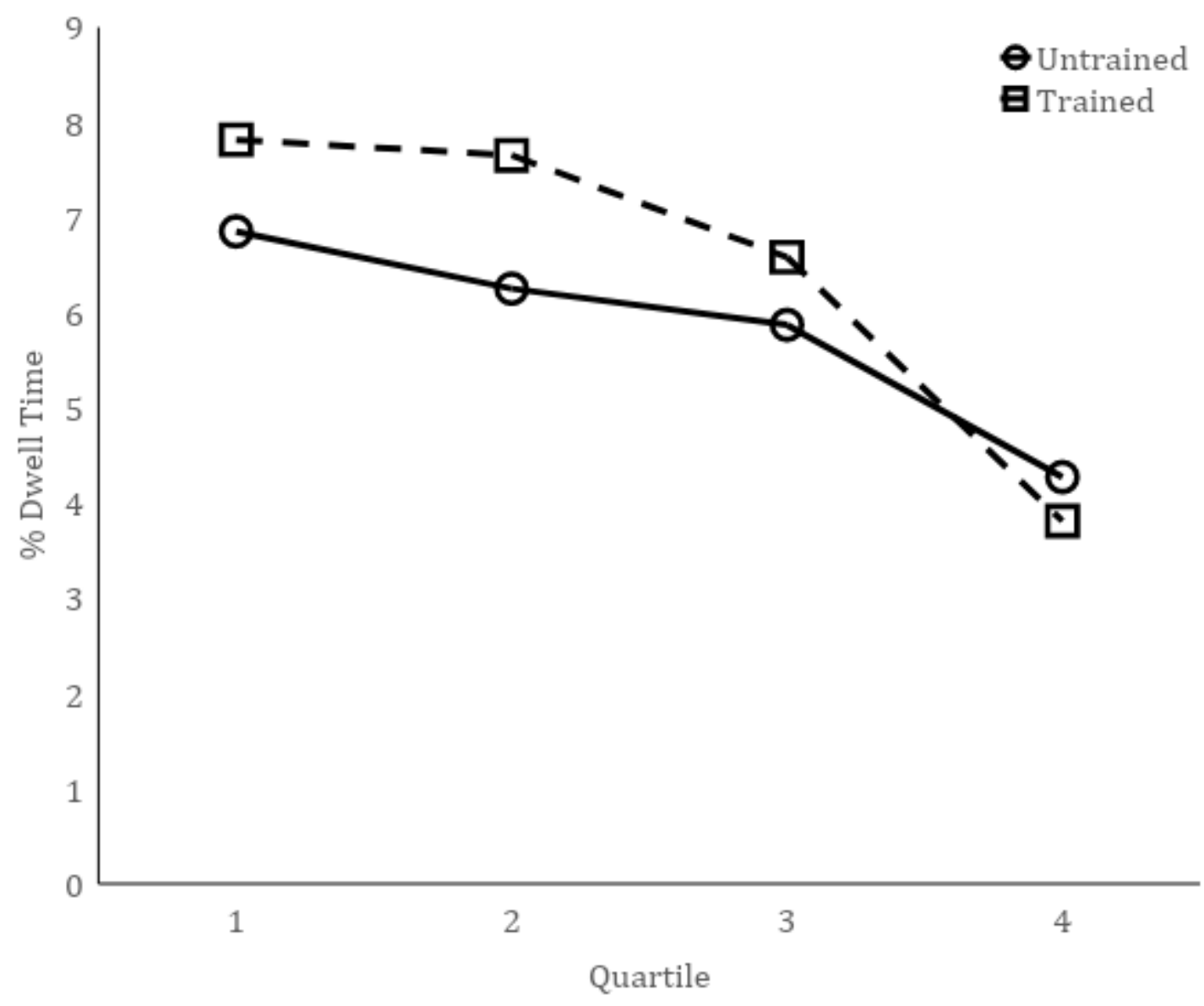

Figure 2: Mean percentage dwell time for trained and untrained participants in all areas of interest across TT distance quartiles. 


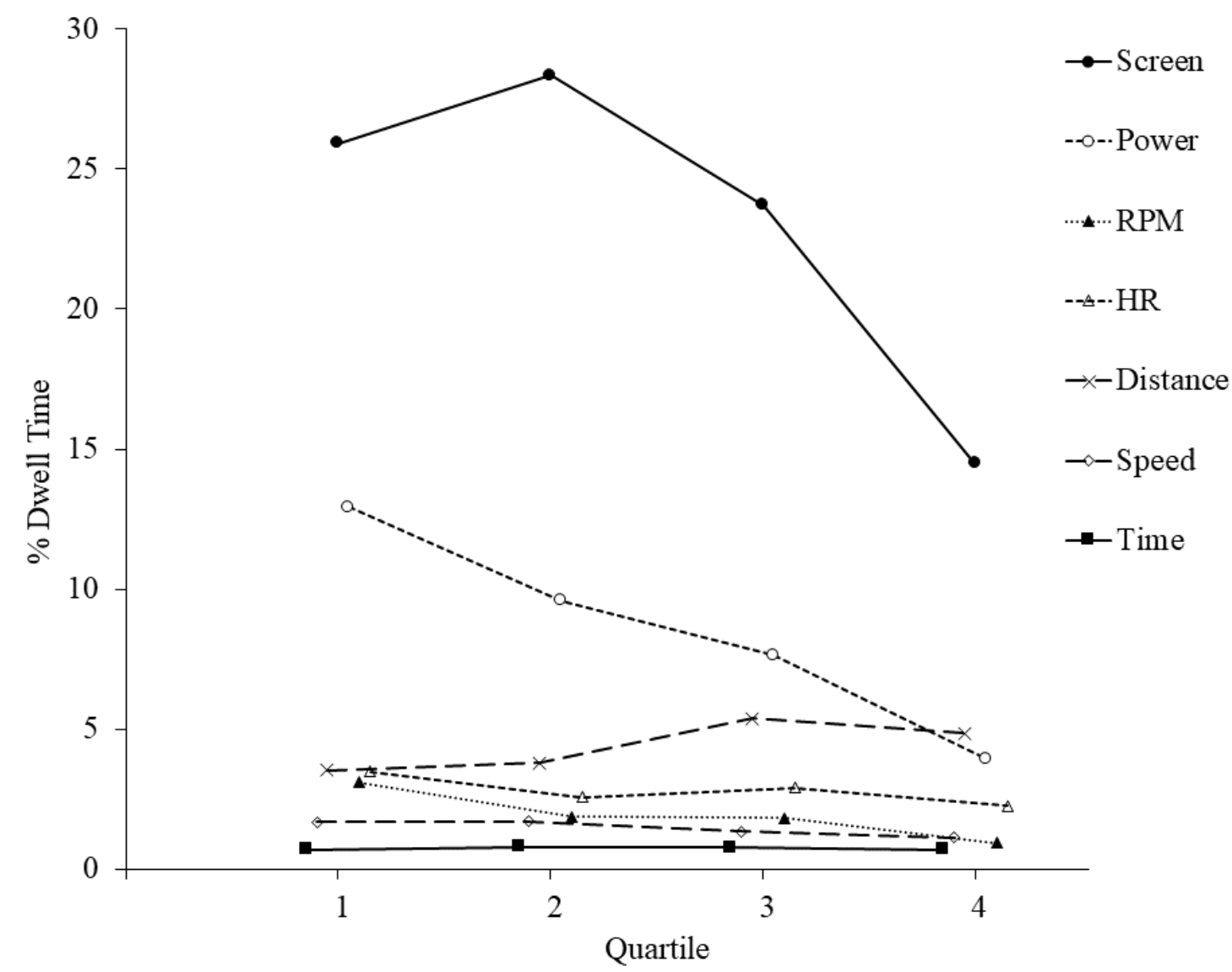

1187

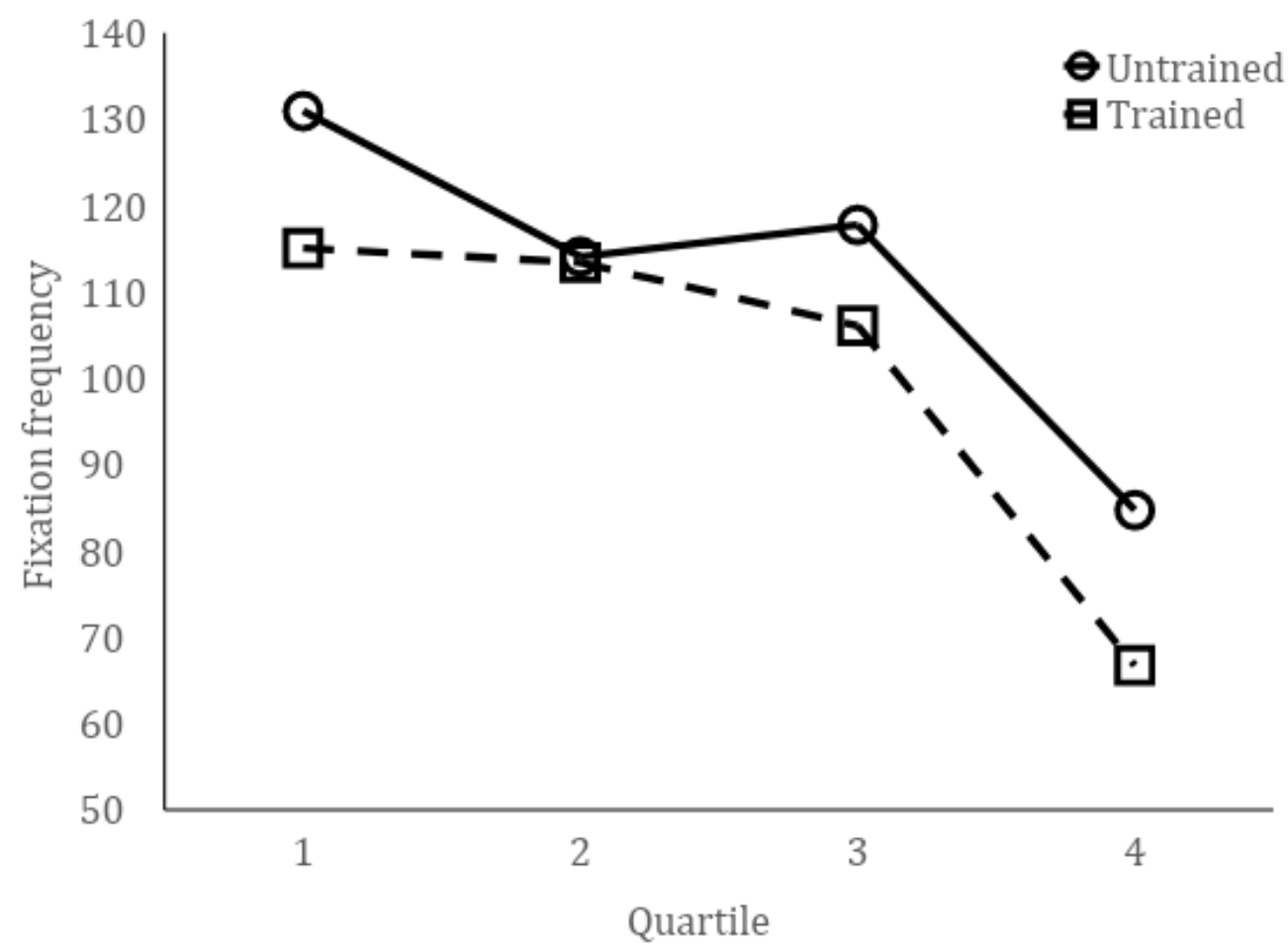


1189

1190

1191

1192

1193

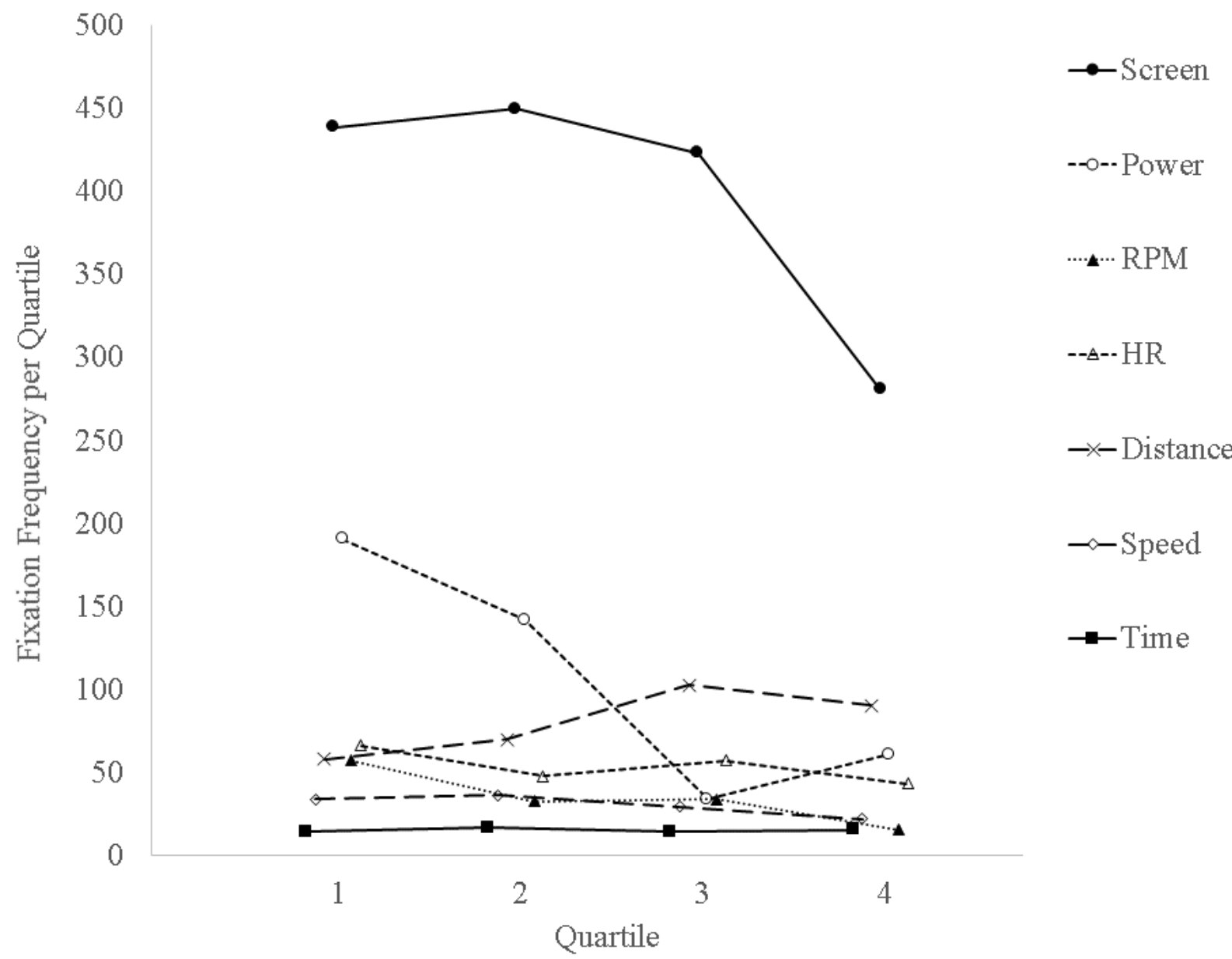

Figure 4: Mean fixation frequency for trained and untrained participants in all areas of interest across TT distance quartiles.

1194

Figure 5: Mean fixation frequency for each area of interest across TT distance quartiles. 


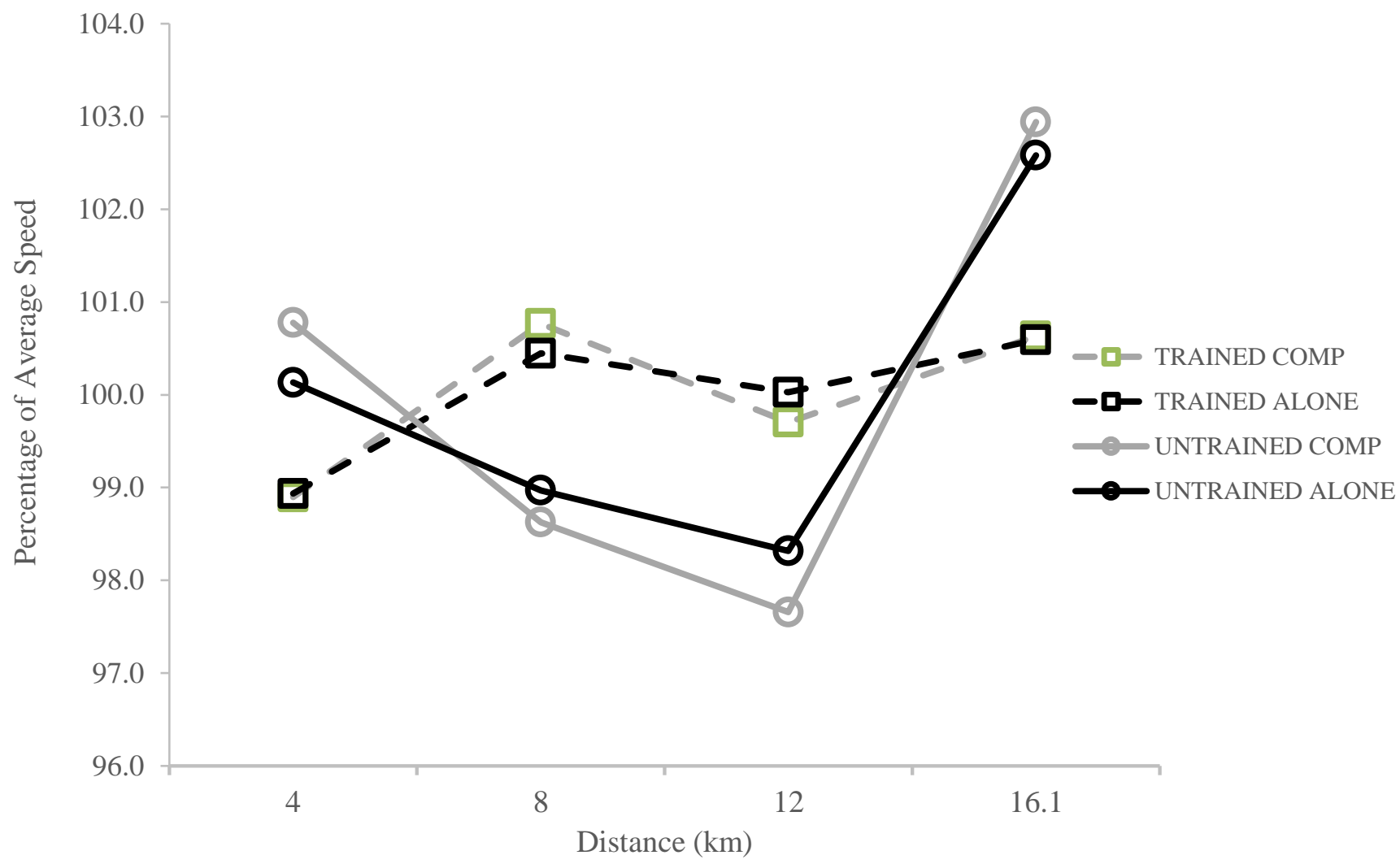

1197 Figure 6: Mean percentage of average speed for trained and untrained groups during alone and 1198 competitor trials.

1199 